\title{
DISTRIBUCIÓN Y PATRONES DE DIVERSIDAD DE LOS AFÓDIDOS EN LA COMUNIDAD DE MADRID (COLEOPTERA, SCARABAEOIDEA, APHODIIDAE, APHODIINAE Y PSAMMODIINAE)
}

\author{
J. Hortal ${ }^{1,2}$, J. M. Lobo ${ }^{1}$ y L. del Rey ${ }^{1}$
}

\begin{abstract}
RESUMEN
En este trabajo se presenta un inventario actualizado de los Aphodiidae (Coleoptera, Scarabaeoidea) de la Comunidad de Madrid. Además, se presentan mapas de la distribución observada y potencial de las 70 especies encontradas en Madrid, así como de la distribución espacial de la riqueza, rareza, y endemicidad potencial en la región. Finalmente, se discute brevemente el origen de los patrones observados.

Palabras clave: escarabajos coprófagos, escarabajos estercoleros, Scarabaeoidea, Aphodiinae, distribución, BIOCLIM, riqueza de especies, rareza, endemicidad, Península Ibérica, Madrid.
\end{abstract}

\begin{abstract}
Spatial distribution and diversity patterns of Aphodids present in Comunidad de Madrid (Coleoptera, Scarabaeoidea, Aphodiidae, Aphodiinae and Psammodiinae)

In this work we present an updated checklist of the Aphodiidae (Coleoptera, Scarabaeoidea) from Comunidad de Madrid (Spain). In addition, the observed and potential distributions of the 70 species found in Madrid are mapped. The potential spatial distributions of species richness, rarity and endemism in this region are also mapped. Finally, we discuss briefly the origin of the observed patterns.
\end{abstract}

Key words: dung beetles, Scarabaeoidea, Aphodiinae, species distribution, BIOCLIM, species richness, rarity, endemism, Iberian Peninsula, Madrid.

\section{Introducción}

Las comunidades de insectos que habitan las heces de los grandes mamíferos son asombrosamente ricas y diversas. Un único lugar puede ser colonizado por más de 200 especies coprófilas (Hanski, 1987), pertenecientes, principalmente, a diferentes familias de coleópteros (Scarabaeidae, Aphodiidae,
Geotrupidae, Hydrophilidae, Staphylinidae, Histeridae, etc.) y dípteros (Muscidae, Calliphoridae, Sarcophagidae, Sepsidae y Sphaerocidae), pero también de himenópteros o ácaros (Martín-Piera \& Lobo, 1995). De entre ellos, los escarabeidos coprófagos (Coleoptera, Scarabaeoidea: Scarabaeidae, Aphodiidae y Geotrupidae) son los que muestran adaptaciones más singulares hacia el consumo de heces, siendo

Departamento de Biodiversidad y Biología Evolutiva. Museo Nacional de Ciencias Naturales (CSIC). C/José Gutiérrez Abascal, 2; Madrid - 28006; Spain

2 Departamento de Ciências Agrárias, CITA-A. Universidade dos Açores. Campus de Angra, Terra-Chã; Angra do Heroísmo - 9701-851 - Terceira (Açores); Portugal 
en las regiones tropicales y templadas, los principales agentes biológicos responsables de la degradación de los excrementos de los grandes herbívoros terrestres (Ridsill-Smith \& Kirk, 1981). Los escarabeidos (superfamilia Scarabaeoidea) constituyen uno de los grupos de coleópteros más característicos y mejor conocidos, no sólo por los entomólogos profesionales y aficionados, sino también por gran parte de la población, especialmente en ambientes rurales. Debido a lo fácil que es observarlos y a su vistosidad, han sido ampliamente colectados y estudiados por entomólogos profesionales y aficionados durante casi tres siglos, por lo que las definiciones de familias y/o subfamilias, así como algunas nociones de su historia evolutiva, están relativamente bien establecidas desde principios del siglo XX (Browne \& Scholtz, 1999). De las más de 20 familias mundiales del grupo Laparosticti (con antenas en forma de maza), seis presentan hábitos coprófagos durante todo su ciclo vital, o, al menos, durante su fase adulta, entre las que destacan Scarabaeidae, Aphodiidae y Geotrupidae (Cambefort, 1991). Estas tres familias constituyen uno de los grupos de insectos más estudiados de la Península Ibérica; desde finales de los años 80, el grado de conocimiento geográfico, faunístico y ecológico se ha incrementado continuamente, por lo que resulta un grupo especialmente adecuado desde el punto de vista del estudio de la biodiversidad, ya que contamos con un importante cuerpo de conocimiento tanto sobre su biología, como sobre sus patrones de distribución (Hortal, 2004).

En este trabajo, realizamos un inventario actualizado de las especies de Aphodiidae (de aquí en adelante, afódidos) presentes en la Comunidad de Madrid. Además, describimos su distribución real y potencial, así como los patrones generales de riqueza de especies, rareza y endemicidad. Para ello, utilizamos los datos recopilados en SCAMAD 2.1, una base de datos faunística de las tres familias coprófagas de Scarabaeoidea, así como la identificación de las especies raras desarrollada por Hortal (2004). Además, utilizamos datos ambientales y una metodología simple con capacidad para elaborar hipótesis de distribución relativamente fiables, a fin de caracterizar los patrones de distribución de la diversidad de afódidos en la Comunidad de Madrid.

\section{Sistemática y características generales de los} Aphodiidae ibéricos

Los estudios filogenéticos existentes en la actualidad permiten sospechar que las especies actualmente adscritas a Scarabaeidae, Aphodiidae y
Geotrupidae constituyen unidades evolutivas muy relacionadas, pero diferentes entre sí (ver Browne \& Scholtz, 1999; Martín-Piera, 2000a; Villalba et al., 2002; Cabrero-Sañudo, 2004; Cabrero-Sañudo \& Zardoya, 2004; Verdú et al., 2004), y que presentan, en general, comportamientos específicos de nidificación y alimentación. Todas están representadas en la Península Ibérica. De las aproximadamente 8.500 especies mundiales de escarabeidos coprófagos (Lobo, 1992a), alrededor de 200 están presentes en la Península Ibérica (Martín-Piera, 2000b; Veiga, 1998; López-Colón, 2000), de las que más de 120 son afódidos.

La gran mayoría de las especies coprófagas de Aphodiidae pertenecen a una sola subfamilia, Aphodiinae, y a un solo género hiperdiverso de amplia distribución, Aphodius (Cambefort \& Hanski, 1991). El debate sobre si varios de los subgéneros de éste deben ser elevados a la categoría de género abierto por Dellacasa et al. (2000) aún no está cerrado (Hortal et al., 2002; Cabrero-Sañudo, 2004; Cabrero-Sañudo \& Zardoya, 2004; J. R. Verdú, comunicación personal). Para evitar problemas derivados de estos cambios taxonómicos, en este trabajo vamos a utilizar la taxonomía utilizada por Veiga (1998) para los Aphodiinae ibéricos, a la espera de que los datos filogenéticos permitan en un futuro próximo adecuar la nomenclatura de las categorías supraespecíficas a utilizar. La otra subfamilia estudiada, Psammodiinae, es mucho menos diversa; en este caso, nos hemos basado en la taxonomía propuesta por Baraud (1992).

La mayoría de los afódidos son habitualmente endocópridos (o moradores, del inglés dwellers; ver Halffter \& Matthews, 1966; Halffter \& Edmonds, 1982; Doube, 1990; Cambefort \& Hanski, 1991), por lo que todo el proceso de desarrollo que va desde la oviposición hasta la pupación, pasando por las distintas fases larvarias, transcurre generalmente en el interior de los excrementos. Su elevado número, tanto en individuos como en especies (unas 110 en la Península; Veiga, 1998), les convierte muchas veces en los principales agentes desintegradores de los excrementos ibéricos (Lobo, 1992a). Además del comportamiento típicamente endocóprido, algunas especies son capaces de construir primitivos nidos en el suelo (p.e. en el subgénero Colobopterus: Aphodius erraticus, Rojewski, 1983), otras presentan estados larvarios radicícolas, y otras incluso presentan comportamiento cleptoparasitos (es decir, aprovechan el momento de la construcción del nido para introducirse o depositar su huevo en las bolas creadas por otras especies; Cambefort \& Hanski, 1991; Martín-Piera \& Lobo, 1993; González-Megías 
\& Sánchez-Piñero, 2003). Aunque Cambefort \& Hanski (1991) consideran este último comportamiento poco significativo, estudios recientes demuestran que puede ser común cuando existen altas densidades de rodadores (Martín-Piera \& Lobo, 1993), e incluso ser un factor importante en la estructuración de las comunidades coprófagas de ambientes mediterráneos semiáridos (González-Megías \& Sánchez-Piñero, 2003), como los presentes en buena parte de la Península Ibérica.

\section{Inventario de los Aphodiidae madrileños}

La información disponible acerca de la distribución de las especies de afódidos en la Comunidad de Madrid y sus alrededores proviene de SCAMAD (Hortal, 2004). Esta base de datos biológica recopila toda la información existente en colecciones de museos, universidades y colecciones privadas, así como la información corológica publicada en la literatura existente sobre las tres familias coprófagas de Scarabaeoidea. SCAMAD consta de 34 campos diferentes, pertenecientes a 5 categorías:

- Campos taxonómicos (Familia, Género, Especie, Autor y Año de descripción),

- Campos corológicos (Topónimo, Municipio y Provincia se refieren al lugar en que se produjo la captura; Altitud localidad y Altitud captura son las altitudes del municipio o localidad y la específica del lugar de captura; UTM completa, UTM $10 \mathrm{~km}$ y UTM propia son, respectivamente, la coordenada UTM con una resolución de $1 \mathrm{~km}^{2}$, la cuadrícula UTM de $10 \mathrm{~km}^{2}$ a la que fue asignada originalmente, y la cuadrícula UTM de $10 \mathrm{~km}^{2}$ a la que realmente pertenece; Latitud y Longitud son las coordenadas UTM a la mayor resolución posible),

- Campos temporales (Día, Mes y Año),

- Campos eco-biológicos (número de ejemplares capturados, Sexo, Alimentación y Hábitat),

- Campos de origen de la información (Método de captura, Colección, Colector, Determinador, Cita bibliográfica, Muestreo, Estación y Trampa).

La versión actual de base de datos (SCAMAD 2.1) incluye información revisada acerca de todos los individuos de la familia Aphodiidae provenientes de tres fuentes. En primer lugar, de los especimenes almacenados en la Colección del Museo Nacional de Ciencias Naturales, en la colección de entomología de la Universidad Complutense de
Madrid, y en las colecciones particulares de J. J. de la Rosa y M. Corra. En segundo lugar, incluye toda la información faunística disponible en la bibliografía sobre la distribución de los afódidos en el centro de la península (ver Hortal, 2004). Finalmente, incluye datos procedentes de campañas de muestreo aún no publicadas, y de diversas capturas manuales no publicadas y realizadas por distintos investigadores (J. Hortal, F. J. Cabrero Sañudo, J. M. Lobo y F. Martín-Piera), así como todos los individuos recolectados durante un muestreo exhaustivo realizado recientemente (19992002) en la Comunidad de Madrid y alrededores (Hortal, 2004; ver también Hortal \& Lobo, 2005).

En total, SCAMAD 2.1 contiene datos acerca de 75 especies diferentes de afódidos. Sin embargo, tras un análisis cuidadoso de las citas más dudosas, se eliminaron 4 de estas especies:

- Aphodius (Chilothorax) brancoi Baraud, 1981. Durante el muestreo de la Comunidad de Madrid se capturaron varios individuos similares a los ejemplares tipo de este taxón en el sur del territorio estudiado; sin embargo, la validez de esta especie (muy similar a A. (Ch.) distinctus) es dudosa (Z. Stebnicka y J.R. Verdú, comunicación personal), por lo que se no se ha considerado en este inventario.

- Aphodius (Chilothorax) sesquivittatus Fairmaire, 1883 y Aphodius (Eupleurus) subterraneus (Linnaeus, 1758), citados por Toribio (1985), pero cuyas identificaciones han sido calificadas como dudosas por Veiga (1998).

- Aphodius (Nimbus) obliteratus Panzer, 1823. Según Veiga (1998), la gran mayoría de los individuos identificados como pertenecientes a esta especie en la Península Ibérica son, en realidad, individuos de $A$. (Nimbus) affinis rozados; aunque este autor cita una captura de El Escorial y otra de El Tiemblo (Ávila) en su colección privada, no hemos incluido esta especie en el inventario de Madrid, a expensas de que nuevos individuos de esta especie, cuyas citas más próximas se encontrarían en Pirineos (según Veiga, 1998), confirmen la existencia de poblaciones en el Sistema Central.

En total, SCAMAD 2.1 contiene información acerca de 116.777 especimenes de afódidos (agrupados en 3,294 registros). El catálogo de Aphodiidae de Madrid queda, por lo tanto, establecido en 70 especies, pertenecientes a dos géneros de Aphodiinae, Aphodius (63 especies) y Heptaulacus (una especie), y a cuatro de Psammodiinae, 
Tabla 1.- Inventario y características de las 70 especies de Aphodiidae existentes en la Comunidad de Madrid. $R$ es el número de registros existentes en SCAMAD 2.1 para cada una de las especies; $N$ el número de individuos registrados hasta la fecha; $C$ es el número de cuadrículas de la malla UTM de $100 \mathrm{~km}^{2}$ en las que ha sido encontrada; Rng es su rango de distribución en el Paleártico Occidental, según las categorías de Lumaret \& Lobo (1996); End son las especies endémicas de la Península Ibérica (categorías de rango entre I a IV); $R h, R d$ y $R r$ son, respectivamente, las especies raras con respecto al hábitat, a su demografía, y a su rango de distribución en Madrid (ver texto y Hortal, 2004). Taxonomía según Veiga (1998) (Aphodiinae) y Baraud (1992) (Psammodiinae).

Table 1.- Checklist and characteristics for the 70 Aphodiidae species present in Comunidad de Madrid. $R$ is the number of database records in SCAMAD 2.1; $N$ is the number of individuals recorded; $C$ is the number of UTM cells of $100 \mathrm{~km}^{2}$ where the species has been recorded; Rng its the distribution range in the Western Palaearctic, following Lumaret \& Lobo (1996) categories; End are the Iberian endemics (range categories from I to IV), and $R h, R d$ and $R r$ are, respectively, the species found to be rare with respect to habitat, demography, and distribution range in Madrid (see text and Hortal, 2004). Systematics according to Veiga (1998) (Aphodiinae) and Baraud (1992) (Psammodiinae).

\begin{tabular}{|c|c|c|c|c|c|c|c|c|}
\hline Especie & $\boldsymbol{R}$ & $N$ & $C$ & Rng & End & $\boldsymbol{R h}$ & $\boldsymbol{R d}$ & $R \boldsymbol{r}$ \\
\hline Aphodius (Acanthobodilus) immundus Creutzer, 1799 & 60 & 205 & 15 & VI & 0 & 0 & 0 & 0 \\
\hline Aphodius (Acrossus) carpetanus Graells, 1847 & 48 & 214 & 12 & II & 1 & 0 & 0 & 0 \\
\hline Aphodius (Acrossus) depressus (Kugelann, 1792) & 30 & 77 & 6 & VI & 0 & 0 & 0 & 0 \\
\hline Aphodius (Acrossus) luridus (Fabricius, 1775) & 34 & 210 & 12 & VI & 0 & 0 & 0 & 0 \\
\hline Aphodius (Acrossus) rufipes (Linnaeus, 1758) & 5 & 6 & 5 & VI & 0 & 1 & 1 & 1 \\
\hline Aphodius (Agolius) bonvouloiri Harold, 1860 & 57 & 1119 & 12 & II & 1 & 0 & 0 & 0 \\
\hline Aphodius (Agrilinus) ater (DeGeer, 1774) & 1 & 2 & 1 & VI & 0 & 1 & 1 & 1 \\
\hline Aphodius (Agrilinus) constans Duftschmid, 1805 & 130 & 1211 & 25 & VI & 0 & 0 & 0 & 0 \\
\hline Aphodius (Agrilinus) ibericus Harold, 1874 & 4 & 32 & 4 & VI & 0 & 1 & 0 & 1 \\
\hline Aphodius (Agrilinus) scybalarius (Fabricius, 1781) & 60 & 228 & 15 & VI & 0 & 0 & 0 & 0 \\
\hline Aphodius (Alocoderus) hydrochaeris (Fabricius, 1798) & 7 & 10 & 4 & VI & 0 & 1 & 1 & 1 \\
\hline Aphodius (Ammoecius) elevatus (Olivier, 1789) & 37 & 115 & 16 & VI & 0 & 0 & 0 & 0 \\
\hline Aphodius (Ammoecius) frigidus (Brisout, 1866) & 26 & 297 & 11 & III & 1 & 0 & 0 & 0 \\
\hline Aphodius (Ammoecius) lusitanicus (Erichson, 1848) & 10 & 58 & 6 & IV & 1 & 1 & 0 & 0 \\
\hline Aphodius (Anomius) annamariae Baraud, 1982 & 91 & 494 & 26 & III & 1 & 0 & 0 & 0 \\
\hline Aphodius (Anomius) castaneus Illiger, 1803 & 39 & 214 & 10 & $\mathrm{~V}$ & 0 & 0 & 0 & 0 \\
\hline Aphodius (Aphodius) conjugatus (Panzer, 1795) & 94 & 660 & 20 & VI & 0 & 0 & 0 & 0 \\
\hline Aphodius (Aphodius) fimetarius (Linnaeus, 1758) & 298 & 2660 & 46 & VI & 0 & 0 & 0 & 0 \\
\hline Aphodius (Aphodius) foetidus (Herbst, 1783) & 232 & 1323 & 45 & VI & 0 & 0 & 0 & 0 \\
\hline Aphodius (Biralus) satellitius (Herbst, 1789) & 49 & 342 & 14 & VI & 0 & 0 & 0 & 0 \\
\hline Aphodius (Bodilus) ictericus (Laichartig, 1781) & 60 & 245 & 23 & VI & 0 & 0 & 0 & 0 \\
\hline Aphodius (Bodilus) longispina Küster, 1854 & 13 & 21 & 8 & $\mathrm{~V}$ & 0 & 0 & 1 & 0 \\
\hline Aphodius (Bodilus) lugens Creutzer, 1799 & 22 & 512 & 12 & VI & 0 & 0 & 0 & 0 \\
\hline Aphodius (Calamosternus) granarius (Linnaeus, 1767) & 161 & 633 & 44 & VI & 0 & 0 & 0 & 0 \\
\hline Aphodius (Calamosternus) mayeri Pilleri, 1953 & 9 & 14 & 5 & VI & 0 & 1 & 1 & 1 \\
\hline Aphodius (Calamosternus) unicolor (Olivier, 1789) & 26 & 620 & 11 & VI & 0 & 0 & 0 & 0 \\
\hline Aphodius (Chilothorax) cervorum Fairmare, 1871 & 9 & 31 & 5 & $\mathrm{~V}$ & 0 & 1 & 0 & 1 \\
\hline Aphodius (Chilothorax) distinctus (O. F. Müller, 1776) & 139 & 5433 & 31 & VI & 0 & 0 & 0 & 0 \\
\hline Aphodius (Chilothorax) lineolatus Illiger, 1803 & 19 & 84 & 9 & VI & 0 & 0 & 0 & 0 \\
\hline Aphodius (Chilothorax) melanostictus W. Schmidt, 1840 & 22 & 193 & 9 & VI & 0 & 0 & 0 & 0 \\
\hline Aphodius (Chilothorax) paykulli Bedel, 1907 & 6 & 20 & 5 & VI & 0 & 1 & 1 & 1 \\
\hline Aphodius (Chilothorax) sticticus (Panzer, 1798) & 24 & 114 & 11 & VI & 0 & 0 & 0 & 0 \\
\hline Aphodius (Colobopterus) erraticus (Linnaeus, 1758) & 161 & 4155 & 34 & VI & 0 & 0 & 0 & 0 \\
\hline Aphodius (Coprimorphus) scrutator (Herbst, 1789) & 84 & 361 & 21 & VI & 0 & 0 & 0 & 0 \\
\hline Aphodius (Esymus) merdarius (Fabricius, 1775) & 62 & 221 & 17 & VI & 0 & 0 & 0 & 0 \\
\hline Aphodius (Esymus) pusillus (Herbst, 1789) & 15 & 142 & 5 & VI & 0 & 0 & 0 & 1 \\
\hline Aphodius (Eudolus) quadriguttatus (Herbst, 1783) & 26 & 55 & 14 & VI & 0 & 0 & 0 & 0 \\
\hline Aphodius (Euorodalus) coenosus (Panzer, 1798) & 28 & 76 & 8 & VI & 0 & 0 & 0 & 0 \\
\hline Aphodius (Euorodalus) paracoenosus Balthasar y Hrubant, 1960 & & 1 & 1 & VI & 0 & 1 & 1 & 1 \\
\hline Aphodius (Euorodalus) tersus Erichson, 1848 & 16 & 60 & 10 & $\mathrm{~V}$ & 0 & 0 & 0 & 0 \\
\hline Aphodius (Labarrus) lividus (Olivier, 1789) & 6 & 5 & 4 & VI & 0 & 1 & 1 & 1 \\
\hline Aphodius (Liothorax) niger (Panzer, 1797) & 31 & 60 & 9 & VI & 0 & 0 & 0 & 0 \\
\hline Aphodius (Mecynodes) striatulus Waltl, 1835 & 1 & 7 & 1 & VI & 0 & 1 & 1 & 1 \\
\hline Aphodius (Melinopterus) abeillei Sietti, 1903 & 2 & 2 & 2 & IV & 1 & 1 & 1 & 1 \\
\hline
\end{tabular}




\begin{tabular}{|c|c|c|c|c|c|c|c|c|}
\hline Especie & $\boldsymbol{R}$ & $N$ & $C$ & Rng & End & $\boldsymbol{R h}$ & $R d$ & $\boldsymbol{R r}$ \\
\hline Aphodius (Melinopterus) consputus Creutzer, 1799 & 17 & 73 & 8 & VI & 0 & 0 & 0 & 0 \\
\hline Aphodius (Melinopterus) prodromus (Brahm, 1790) & 53 & 687 & 26 & VI & 0 & 0 & 0 & 0 \\
\hline Aphodius (Melinopterus) reyi Reitter, 1892 & 8 & 31 & 8 & $\mathrm{~V}$ & 0 & 1 & 0 & 0 \\
\hline Aphodius (Melinopterus) sphacelatus (Panzer, 1798) & 178 & 8108 & 32 & VI & 0 & 0 & 0 & 0 \\
\hline Aphodius (Melinopterus) tingens Reitter, 1892 & 20 & 189 & 10 & VI & 0 & 0 & 0 & 0 \\
\hline Aphodius (Nialus) varians Duftschmid, 1805 & 10 & 23 & 6 & VI & 0 & 1 & 0 & 0 \\
\hline Aphodius (Nimbus) affinis Panzer, 1823 & 172 & 77994 & 31 & VI & 0 & 0 & 0 & 0 \\
\hline Aphodius (Nimbus) contaminatus (Herbst, 1783) & 109 & 4560 & 24 & VI & 0 & 0 & 0 & 0 \\
\hline Aphodius (Nobius) bonnairei Reitter, 1892 & 16 & 118 & 9 & $\mathrm{~V}$ & 0 & 0 & 0 & 0 \\
\hline Aphodius (Otophorus) haemorrhoidalis (Linnaeus, 1758) & 138 & 895 & 23 & VI & 0 & 0 & 0 & 0 \\
\hline Aphodius (Phalacronothus) putoni Reitter, 1894 & 19 & 43 & 8 & III & 1 & 0 & 0 & 0 \\
\hline Aphodius (Phalacronothus) quadrimaculatus (Linnaeus, 1761) & 16 & 81 & 12 & VI & 0 & 0 & 0 & 0 \\
\hline Aphodius (Plagiogonus) nanus Fairmare, 1860 & 2 & 3 & 2 & VI & 0 & 1 & 1 & 1 \\
\hline Aphodius (Planolinus) borealis Gyllenhal, 1827 & 14 & 23 & 6 & VI & 0 & 0 & 0 & 0 \\
\hline Aphodius (Planolinus) uliginosus Hardy, 1847 & 4 & 5 & 2 & VI & 0 & 1 & 1 & 1 \\
\hline Aphodius (Sigorus) porcus (Fabricius, 1792) & 7 & 23 & 5 & VI & 0 & 1 & 0 & 1 \\
\hline Aphodius (Subrinus) sturmi Harold, 1870 & 46 & 238 & 11 & VI & 0 & 0 & 0 & 0 \\
\hline Aphodius (Teuchestes) fossor (Linnaeus, 1758) & 96 & 672 & 17 & VI & 0 & 0 & 0 & 0 \\
\hline Aphodius (Trichonotulus) scrofa (Fabricius, 1787) & 60 & 267 & 16 & VI & 0 & 0 & 0 & 0 \\
\hline Heptaulacus testudinarius (Fabricius, 1775) & 11 & 21 & 7 & VI & 0 & 0 & 1 & 0 \\
\hline Platytomus tibialis (Fabricius, 1798) & 2 & 5 & 1 & VI & 0 & 1 & 1 & 1 \\
\hline Pleurophorus caesus (Creutzer, 1796) & 20 & 31 & 10 & VI & 0 & 0 & 0 & 0 \\
\hline Pleurophorus mediterranicus Pittino \& Mariani, 1986 & 4 & 38 & 2 & VI & 0 & 1 & 0 & 1 \\
\hline Psammodius laevipennis Costa, 1844 & 5 & 92 & 3 & VI & 0 & 1 & 0 & 1 \\
\hline Rhyssemus algiricus Lucas, 1846 & 7 & 9 & 4 & VI & 0 & 1 & 1 & 1 \\
\hline Rhyssemus germanus (Linnaeus, 1767) & 5 & 6 & 3 & VI & 0 & 1 & 1 & 1 \\
\hline
\end{tabular}

Platytomus (una especie), Pleurophorus (dos especies), Psammodius (una especie) y Rhyssemus (dos especies) (ver Tabla 1). Tras el muestreo intensivo llevado a cabo en Madrid, el inventario de las especies que forman parte de la fauna de escarabeidos coprófagos de Madrid puede considerarse prácticamente completo. La única especie nueva para Madrid encontrada durante dicho muestreo es Aphodius (Agrilinus) ater, de la que se capturaron dos individuos en la subida al Puerto de La Puebla (1520 m., 30TVL5944, 26-28 de Octubre de 2000; ver Hortal, 2004). Este resultado no es extraño, ya que el esfuerzo de muestreo realizado en Madrid antes de 1999 ha sido lo suficientemente importante como para inventariar prácticamente todas las especies presentes en ella (ver Hortal, 2004), aunque el conocimiento de la distribución espacial de estas especies siga siendo escaso.

\section{Distribución observada y potencial}

Para cada una de las especies de afódidos se ha realizado un mapa de distribución que reúne sus citas geográficas dentro de una región de $160 \mathrm{~km}$ de largo por $170 \mathrm{~km}$ de ancho $\left(27.200 \mathrm{~km}^{2}\right)$ la cual comprende todo el territorio de la Comunidad de Madrid y, parcialmente, el de las provincias limítrofes de Ávila, Cuenca, Guadalajara, Segovia, Toledo y Valladolid. Los cuatro vértices de este área son los correspondientes a las cuadrículas UTM de $100 \mathrm{~km}^{2}$ 30TUL56, 30TWL06, 30SUK60 y 30SWK00. La resolución utilizada para la información corológica fue de $1 \mathrm{~km}$, asignando como coordenadas geográficas las del centroide de la localidad o de la cuadrícula UTM de $100 \mathrm{~km}^{2}$ cuando no existía suficiente precisión en la cita.

La estimación de la distribución potencial de cada una de las especies se realizó reconociendo el conjunto de localidades con condiciones ambientales similares a las existentes en los puntos de presencia conocidos (ver, por ejemplo, Busby, 1986). Calculando los valores máximos y mínimos de cuatro variables climáticas: precipitación total anual, precipitación durante el estío (meses Junio, Julio y Agosto), temperatura máxima del mes más calido (Julio) y temperatura mínima del mes más frío (Enero), la hipótesis de distribución se determinó estableciendo como lugares potencialmente favorables el conjunto de las cuadrículas de $1 \mathrm{~km}^{2}$ con valores climáticos situados entre ambos extremos. La cartografía climática fue proporcionada por el 
Instituto Nacional de Meteorología, siendo elaborada mediante un Sistema de Información Geográfico (Idrisi Kilimanjaro; Clark Labs., 2003).

A continuación se presentan las distribuciones observadas y potenciales de las 70 especies de afódidos en la Comunidad de Madrid (Figura 1).

\section{Patrón de variación geográfica de la diversidad}

La diversidad biológica es un atributo complejo que no puede resumirse mediante una única medida (Gaston, 1996). Por ello, en este caso, hemos utilizado las distribuciones potenciales de las especies de afódidos para caracterizar tres aspectos diferentes de la biodiversidad: riqueza, rareza y endemicidad. En el caso de la riqueza, hemos superpuesto las distribuciones potenciales de todas las especies y para estimar la rareza y endemicidad, hemos calculado el número de especies raras y endémicas en cada punto del territorio analizado (ver Figura 2).

Como el factor clave en el estudio de la rareza es evaluar la importancia de las especies raras en los ensamblajes, es necesario identificar la rareza teniendo en cuenta todas las especies del grupo funcional estudiado. En este caso, los escarabeidos coprófagos constituyen un grupo homogéneo de cara a la colonización y degradación de las heces de grandes mamíferos. Por ello, hemos considerado como especies raras a las identificadas por Hortal (2004) a partir los datos para las tres familias coprófagas de Scarabaeoidea. En ese trabajo, las especies raras se calcularon como el primer cuartil de las especies más raras (Gaston, 1994) en función de tres categorías diferentes: Rareza de hábitat $(R h$; grado de especificidad de hábitat; Rabinowitz et al., 1986), Rareza demográfica ( $R d$; especies con pocos individuos o difíciles de colectar; Rabinowitz et al., 1986; Gaston, 1994) y Rareza de rango (Rr; amplitud de la distribución en el territorio de Madrid). En total, 25 especies pertenecen a una o varias de las categorías de rareza; 22 de ellas eran raras por hábitat, 16 raras demográficamente, y 20 raras en función de su rango (Tabla 1). Es de resaltar que la mayoría de las especies de Psammodiinae (5 de 6) pertenecen a alguna categoría de rareza, mientras que dentro de los Aphodiinae las especies raras se encuentran distribuidas entre diferentes subgéneros.

El concepto de endemicidad como tal constituye en realidad una manifestación de la rareza en el rango geográfico. Mientras que los tres tipos anteriores de rareza pueden ser una expresión de la especificidad ecológica de las especies a pequeña escala, la endemicidad a gran escala puede ser la expresión de la influencia de eventos históricos únicos como determinantes de la distribución actual de las especies (Brown \& Lomolino, 1998). El grado de endemicidad de las especies se determina partir de la amplitud de su distribución geográfica (Gaston, 1994), siendo las especies tanto más endémicas cuanto más localizada sea su presencia. Lumaret y Lobo (1996) asignaron las especies coprófagas de Scarabaeoidea del Paleártico Occidental (es decir, las pertenecientes a las tres familias estudiadas aquí) a seis categorías de endemicidad (localización) diferentes, en función de la proporción entre el tamaño de su rango y la extensión total de esta región biogeográfica. Otros estudios han utilizado estas mismas categorías para caracterizar las especies de estas familias aún no descritas en el Paleártico occidental (CabreroSañudo \& Lobo, 2003), así como para caracterizar la endemicidad de los Scarabaeoidea Australianos (Allsopp, 1999). Hemos tomado, pues, los grados de localización para cada especie utilizados por Cabrero-Sañudo y Lobo (2003; una actualización de los utilizados por Lumaret \& Lobo, 1996), como una medida de la endemicidad de dicha especie. Al igual que Lumaret \& Lobo (1996), hemos considerado como endémicas las especies con rangos comprendidos en las categorías I a IV, es decir, a aquellas con rangos inferiores a $6 \times 10^{5} \mathrm{~km}^{2}$, un tamaño similar al de la Península Ibérica $\left(5.8 \times 10^{5}\right.$ $\mathrm{km}^{2}$ ). En total, tan sólo siete especies son consideradas endémicas según este criterio (ver Tabla 1).

La riqueza potencial de especies oscila entre menos de diez y más de 60 especies por $\mathrm{km}^{2}$ (Figura 2). En general, la riqueza es elevada en una amplia banda de altitudes medias y altas de la Sierra, y baja en las áreas más secas y cálidas de los valles del Tajo y del Alberche, y en las áreas más elevadas de la cuerda serrana. El número de especies raras por $\mathrm{km}^{2}$ varía entre 0 y 16 especies, y presenta un patrón geográfico similar al de la riqueza (coeficiente de correlación de Spearman, $R s=$ $0.965, p<0.001$ ), aunque la banda en la que se solapan el máximo de especies raras sea mucho más estrecha (Figura 2). La endemicidad, en cambio, varía entre 0 y 6 especies endémicas por $\mathrm{km}^{2}$. En este caso, el patrón geográfico, a pesar de ser bastante similar a los dos anteriores (correlación con riqueza: $R s=0.881, p<0.001$; correlación con rareza: $R s=0.818, p<0.001)$, presenta ciertas particularidades, encontrándose el número máximo de especies endémicas en gran parte de las áreas más elevadas de la Sierra (Figura 2).

Durante los últimos años, diversos trabajos han estudiado los factores relacionados con variación 


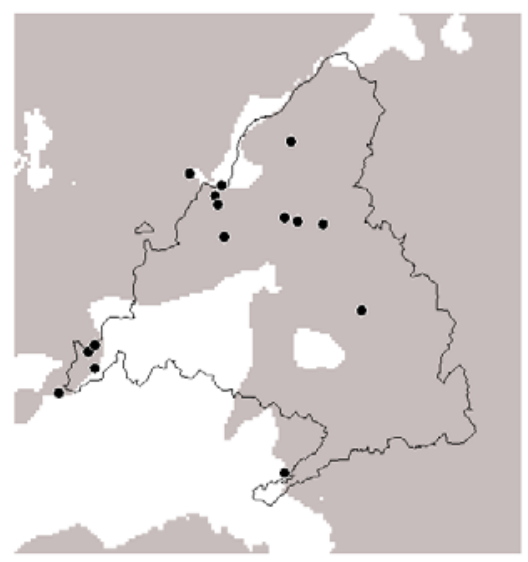

Aphodius (Acanthobodilus ) immundus

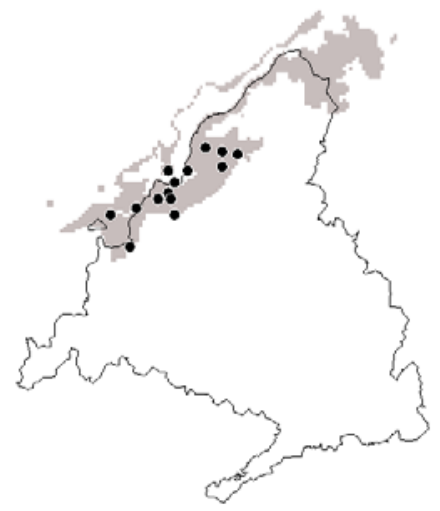

Aphodius ( Acrossus) depressus

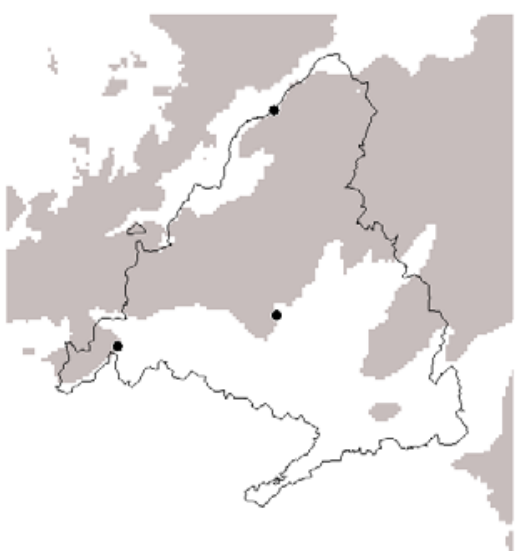

Aphodius (Acrossus) rufipes

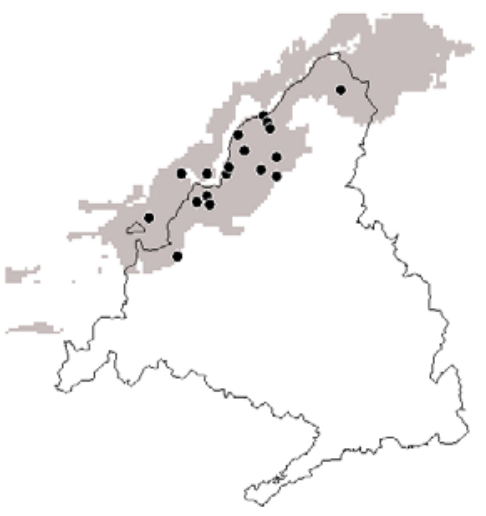

Aphodius (Acrossus) carpetanus

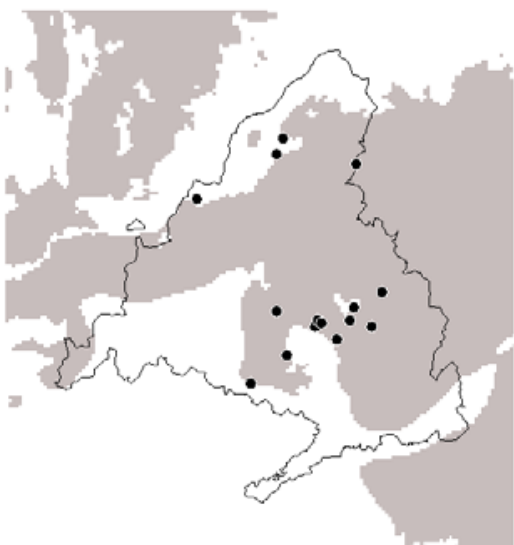

Aphodius (Acrossus) luridus

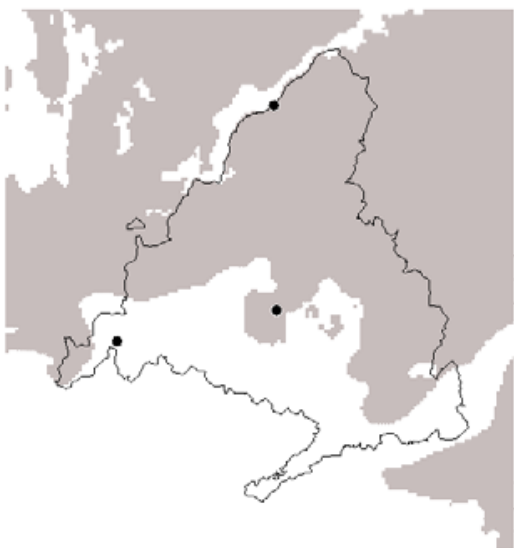

Aphodius (Agolius) bonvouloiri

Fig. 1.- Mapas de distribución observada y potencial en Madrid y alrededores de las 70 especies de Aphodiidae registradas en SCAMAD 2.1. Los puntos son las localidades donde se ha encontrado a cada especie, y las áreas grises su distribución potencial estimada mediante BIOCLIM (ver texto).

Fig. 1.- Observed and potential distribution maps at Madrid and surrounding areas for the 70 Aphodiidae species recorded in SCAMAD 2.1. Points are the localities where each species has been found, and grey areas are their potential distributions estimated with BIOCLIM (see text). 


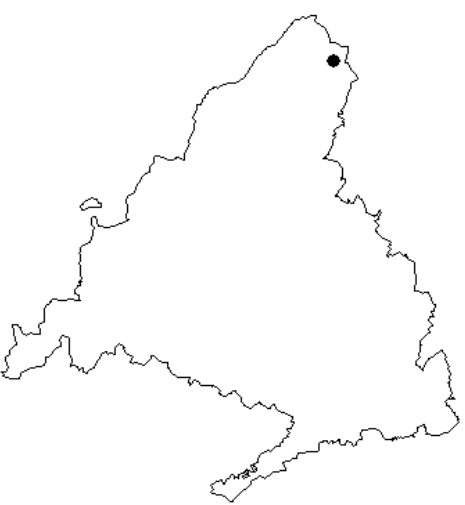

Aphodius (Agrilinus) ater

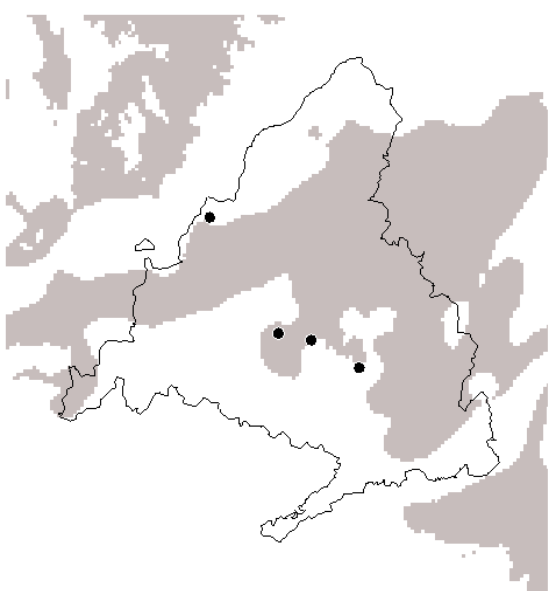

Aphodius (Agrilinus) ibericus

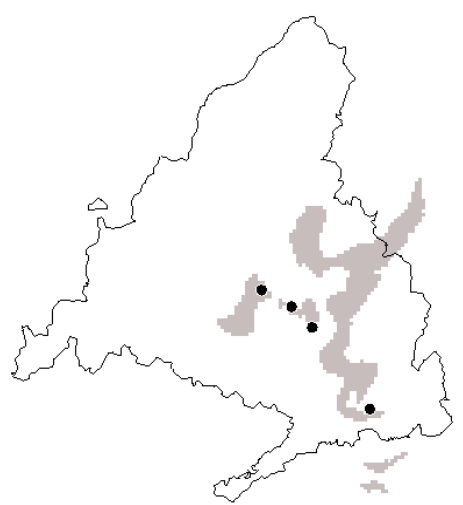

Aphodius (Alocoderus) hydrochaeris

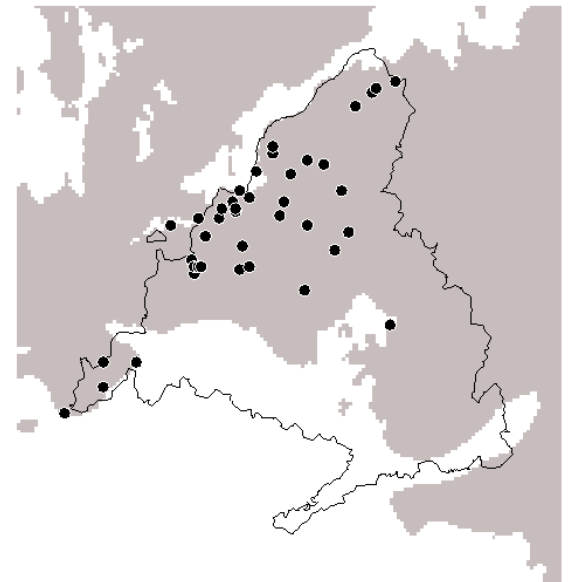

Aphodius (Agrilinus) constants

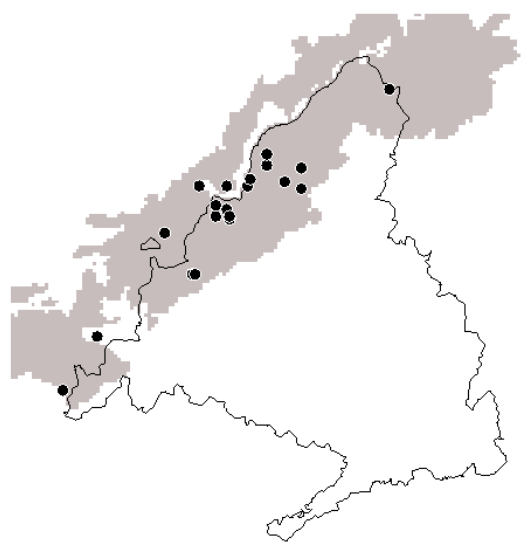

Aphodius (Agrilinus) scybalarius

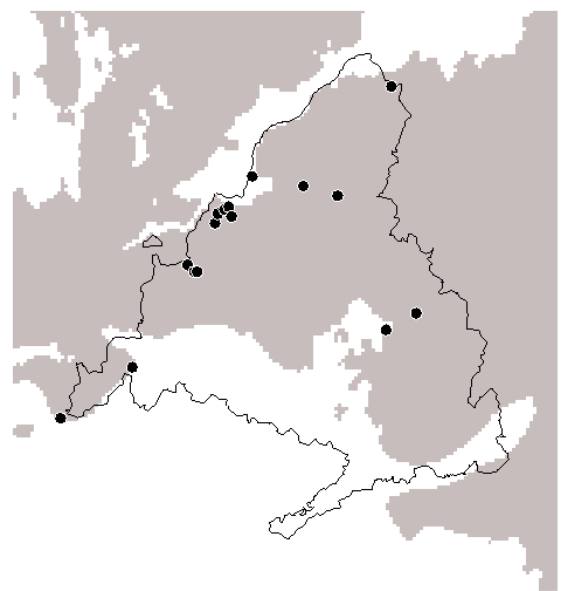

Aphodius (Ammoecius) elevatus

Fig. 1.-Cont 


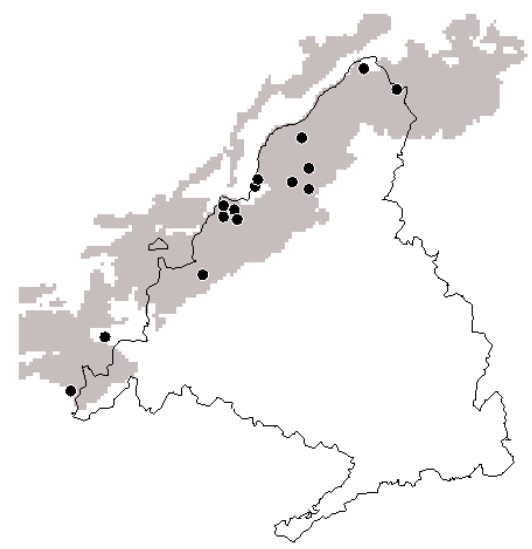

Aphodius (Ammoecius) frigidus

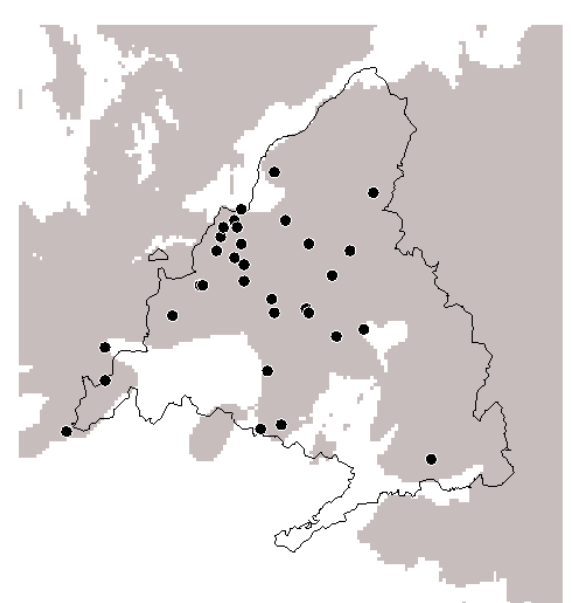

Aphodius (Anomius) annamariae

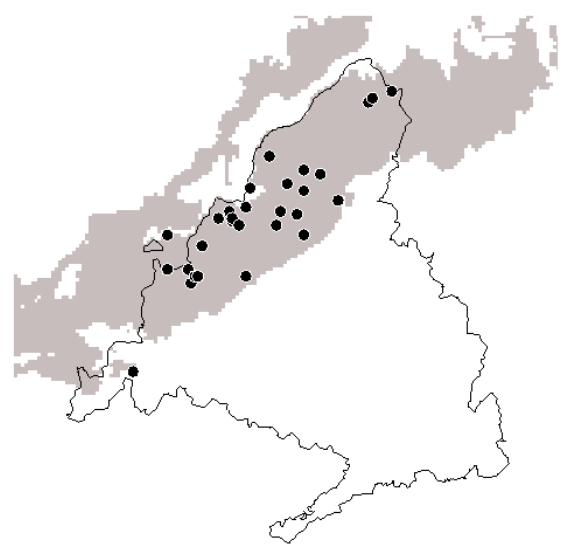

Aphodius (Aphodius) conjugatus

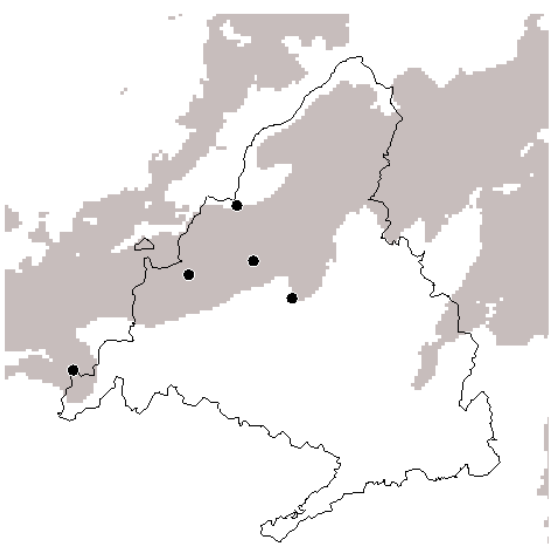

Aphodius (Ammoecius) lusitanicus

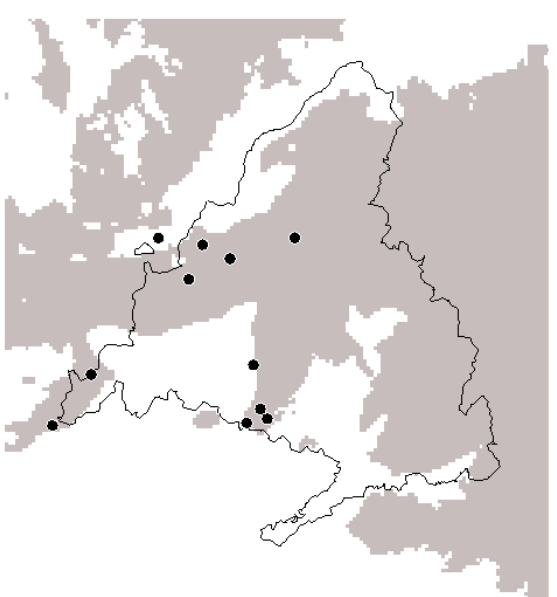

Aphodius (Anomius) castaneus

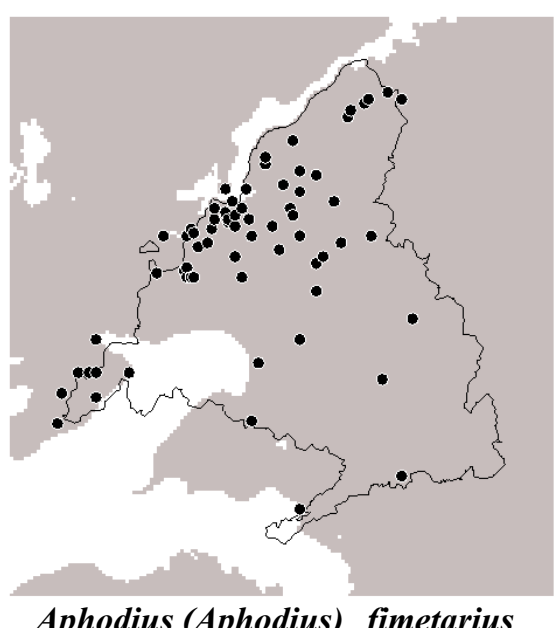

Aphodius (Aphodius) fimetarius

Fig. 1.-Cont. 


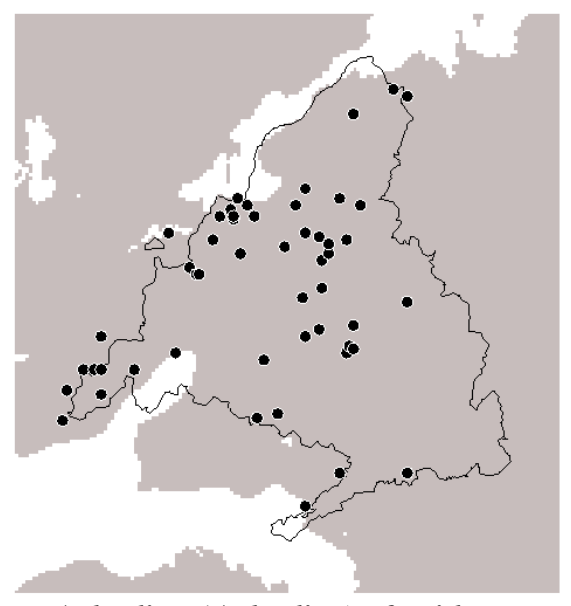

Aphodius (Aphodius) foetidus

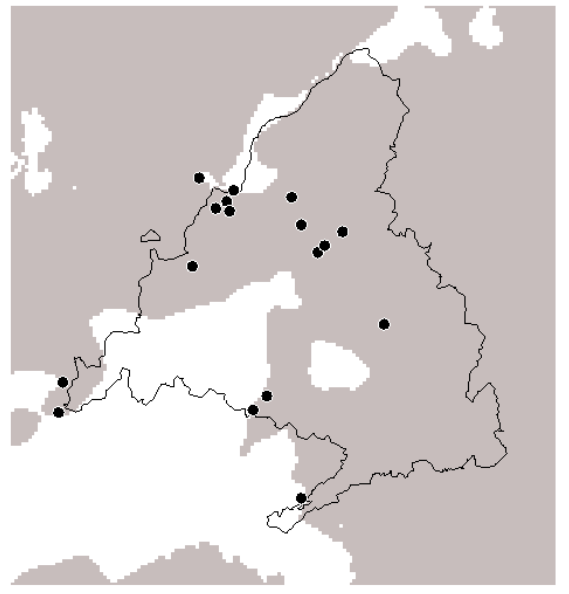

Aphodius (Bodilus) ictericus

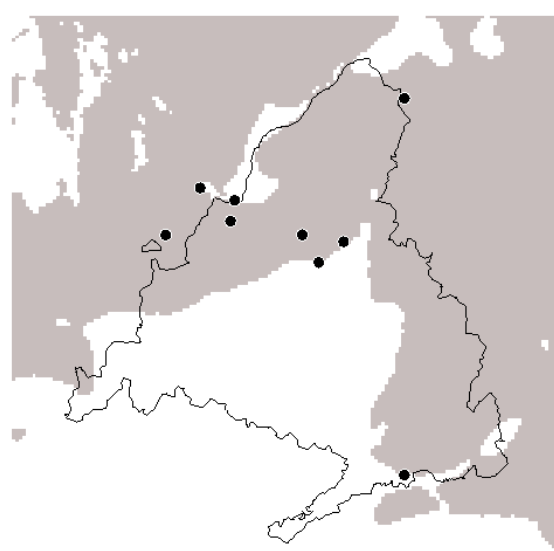

Aphodius (Bodilus) lugens

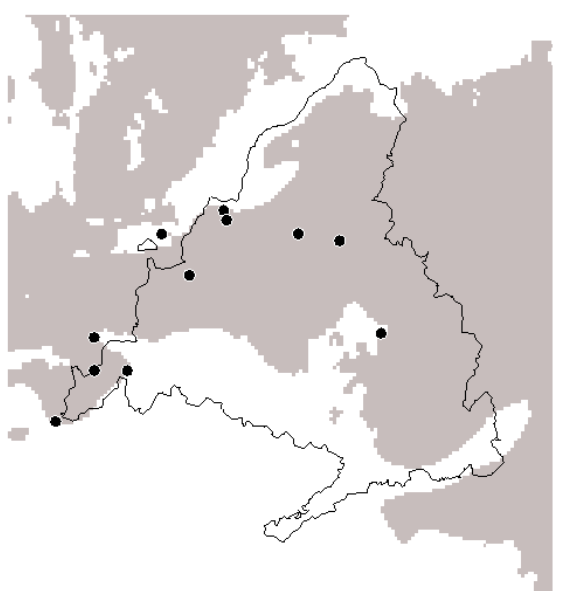

Aphodius (Biralus) satellitius

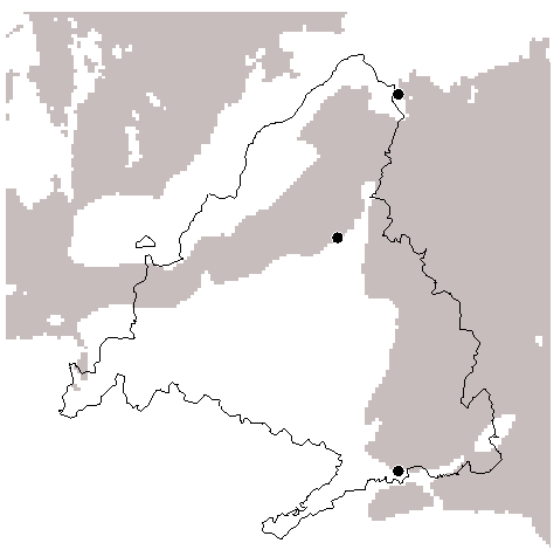

Aphodius (Bodilus) longispina

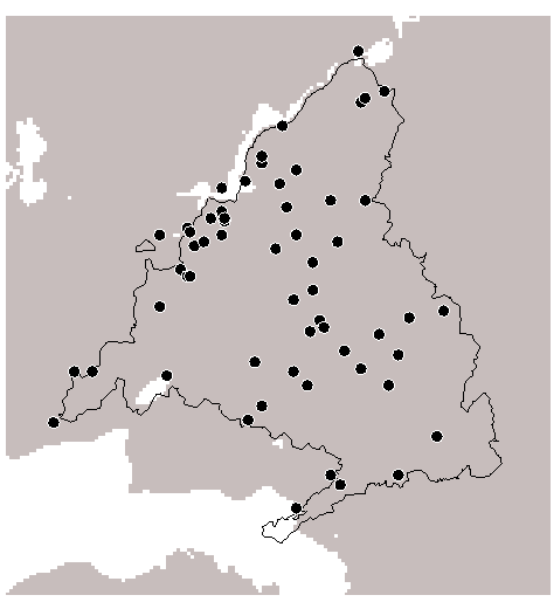

Aphodius (Calamosternus) granarius

Fig. 1.-Cont. 


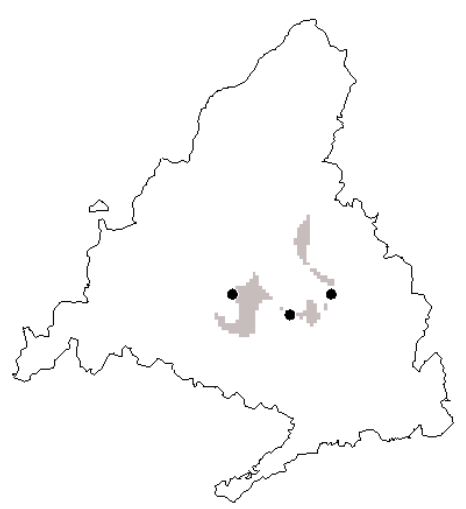

Aphodius ( Calamosternus) mayeri

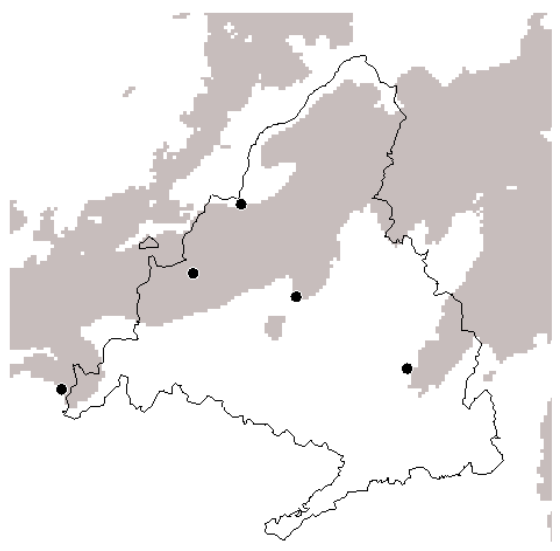

Aphodius (Chilothorax) cervorum

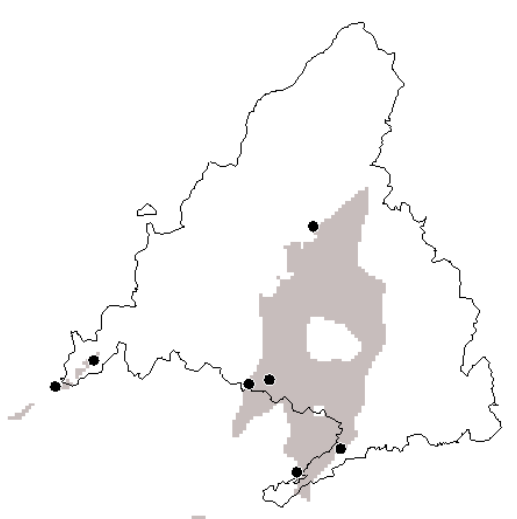

Aphodius (Chilothorax) lineolatus

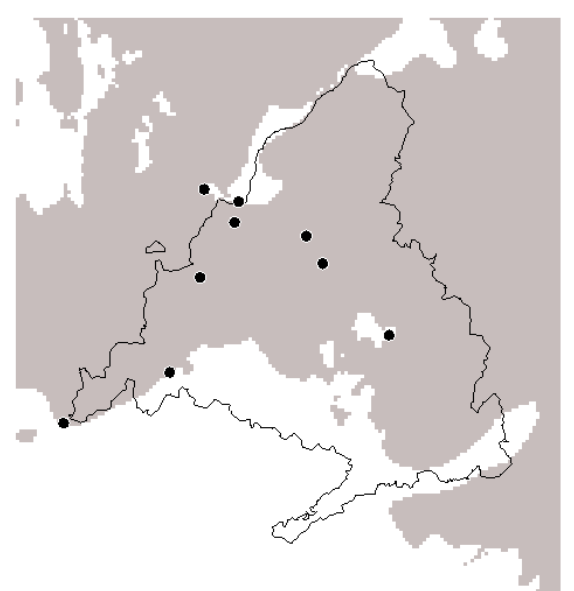

Aphodius (Calamosternus) unicolor
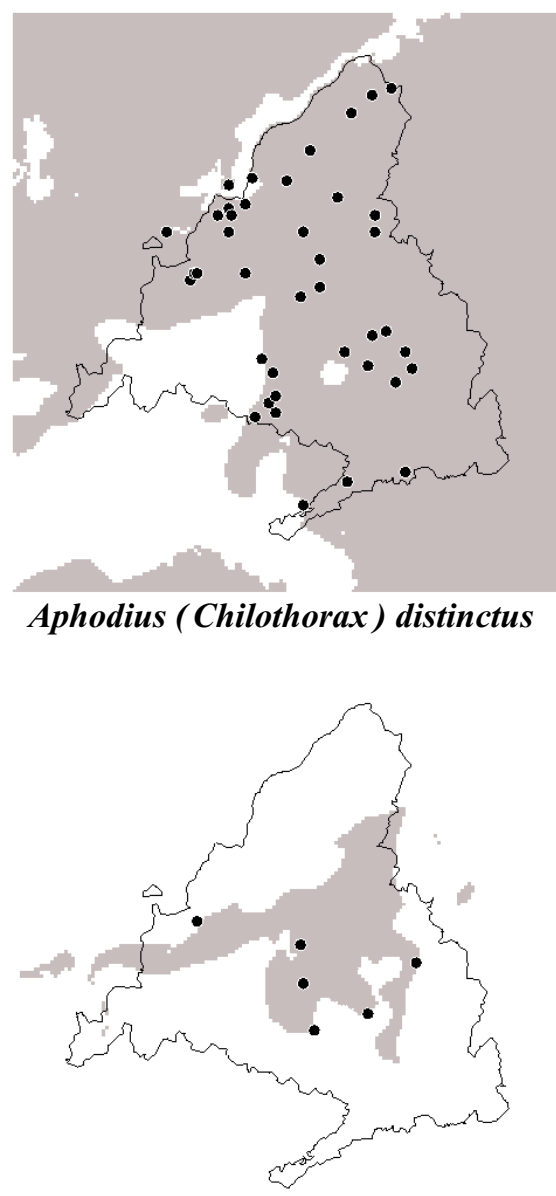

Aphodius (Chilothorax) melanostictus

Fig. 1.-Cont. 


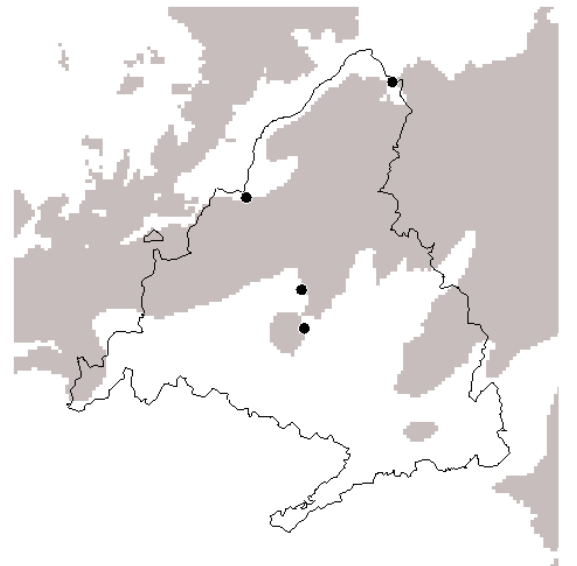

Aphodius (Chilothorax) paykulli

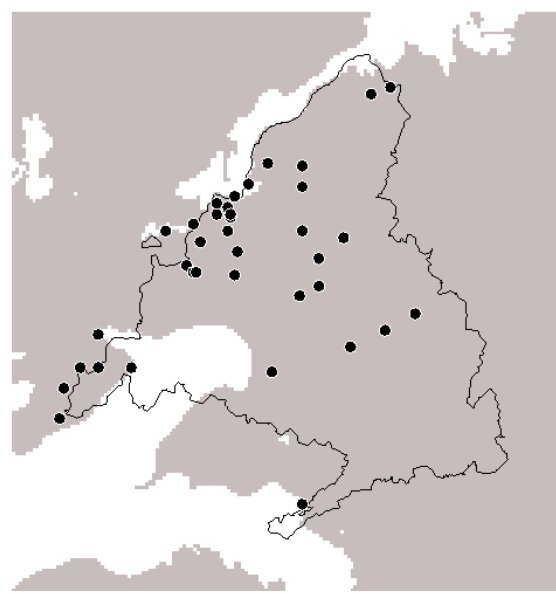

Aphodius (Colobopterus) erraticus

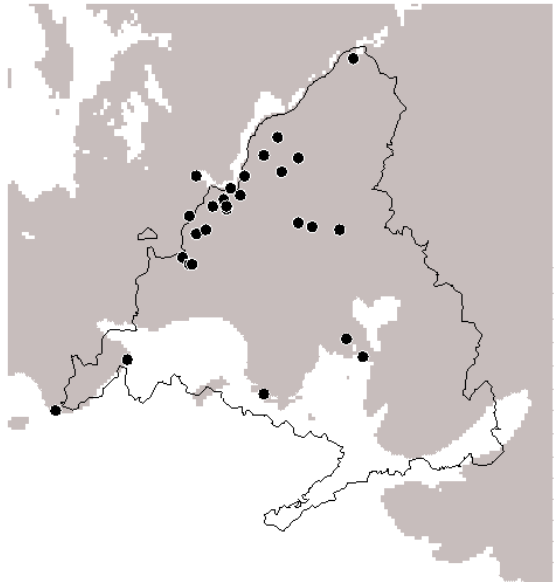

Aphodius (Esymus) merdarius

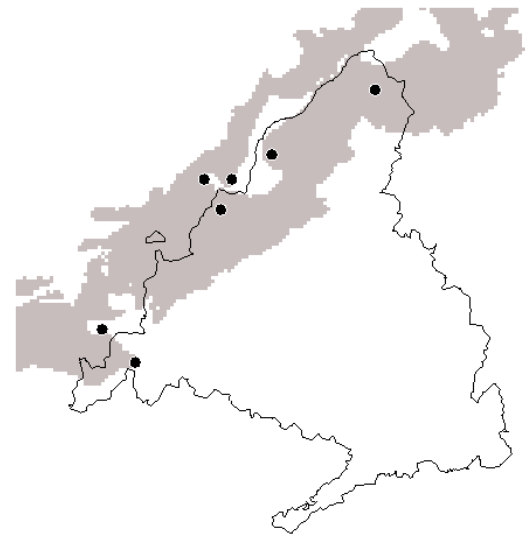

Aphodius (Chilothorax) sticticus

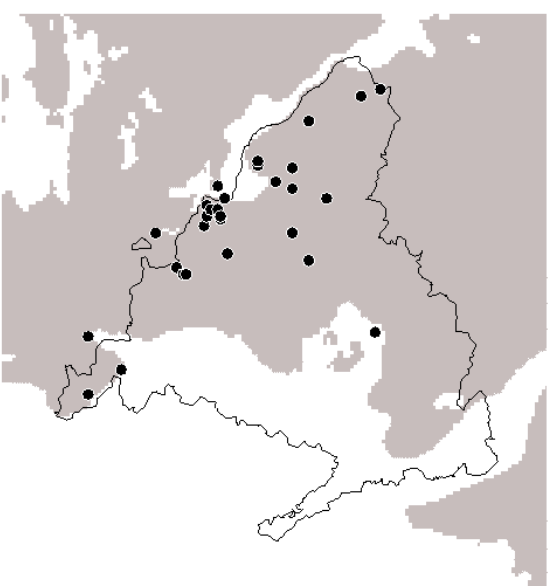

Aphodius (Coprimorphus) scrutator

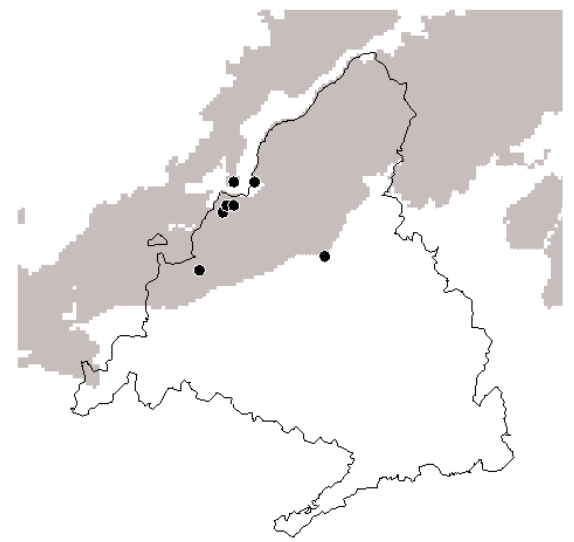

Aphodius (Esymus) pusillus

Fig. 1.- Cont 


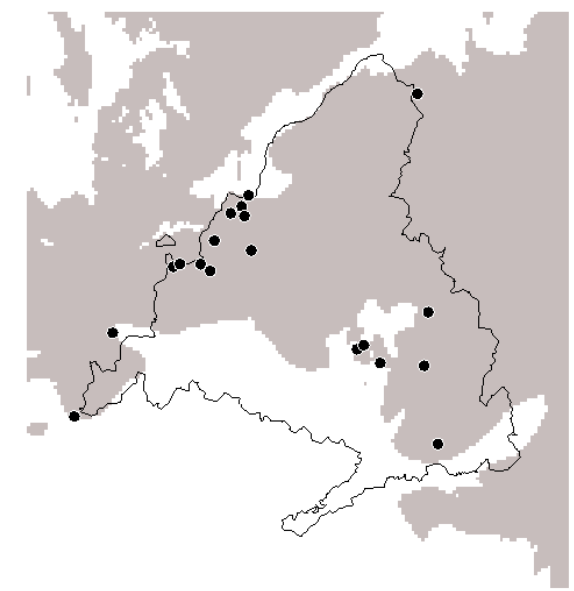

Aphodius ( Eudolus) quadriguttatus

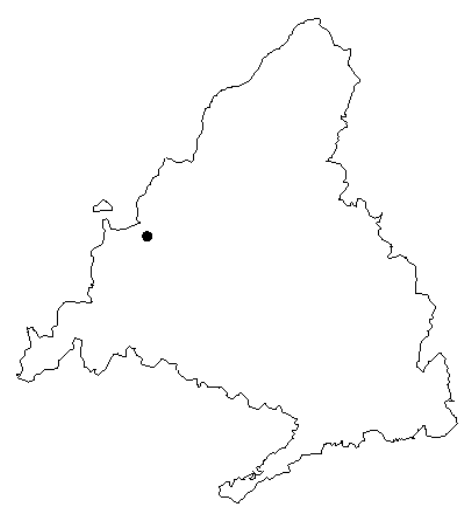

Aphodius (Euorodalus) paracoenosus

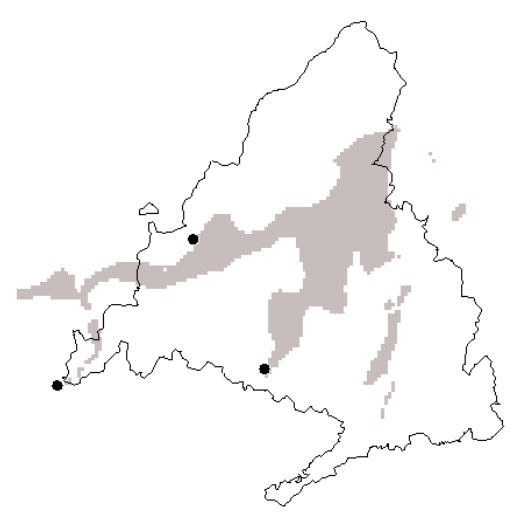

Aphodius (Labarrus) lividus

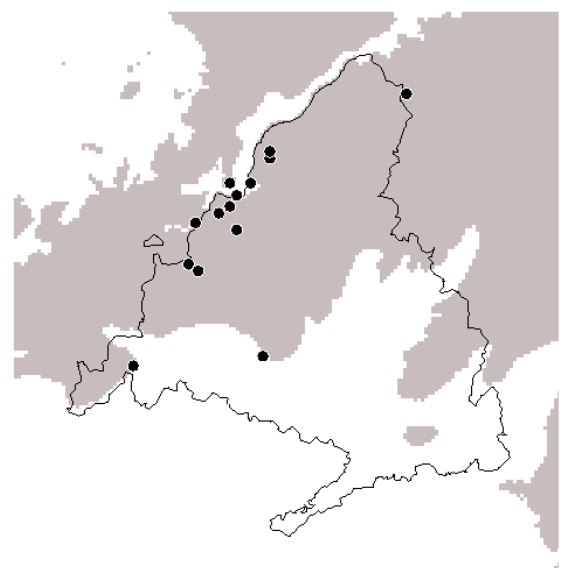

Aphodius (Euorodalus) coenosus

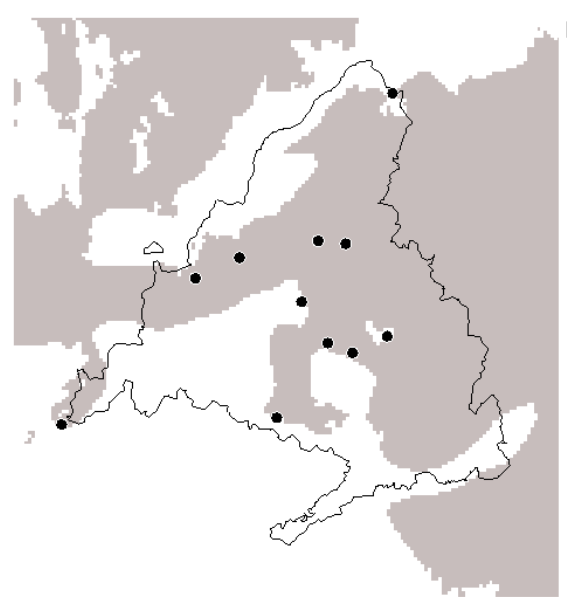

Aphodius (Euorodalus) tersus

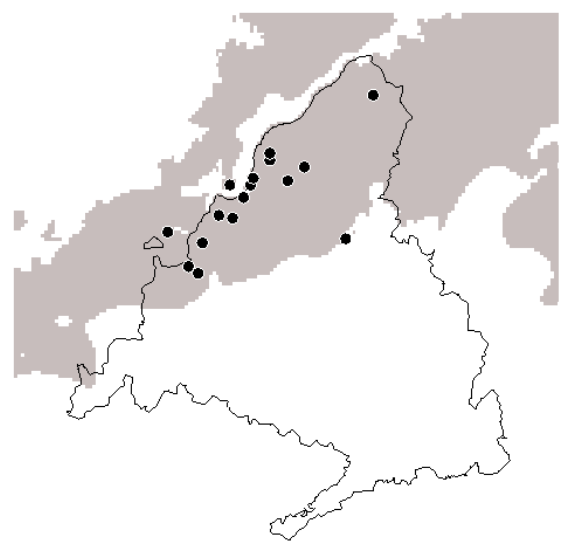

Aphodius (Liothorax) niger

Fig. 1.- Cont. 


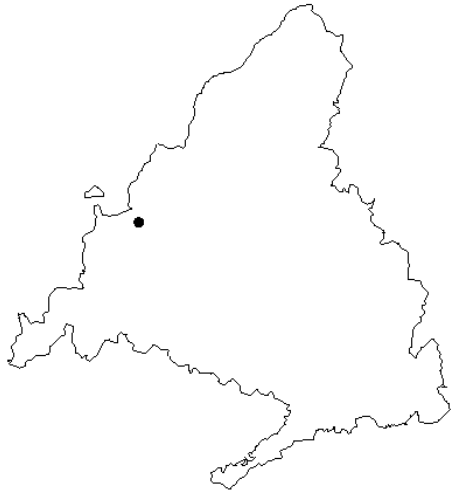

Aphodius (Mecynodes) striatulus

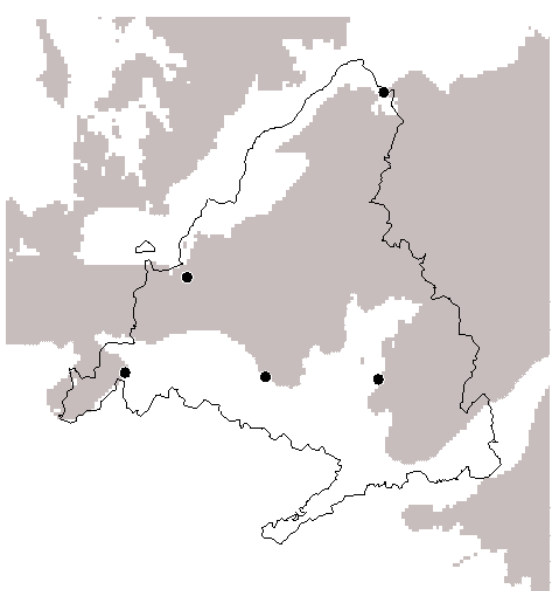

Aphodius (Melinopterus) consputus

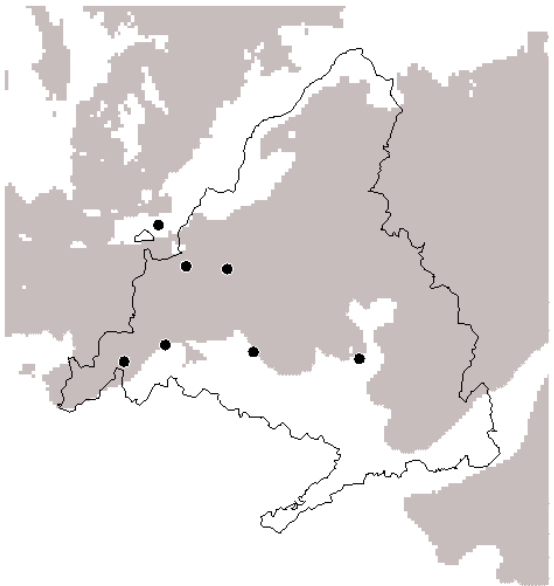

Aphodius (Melinopterus) reyi

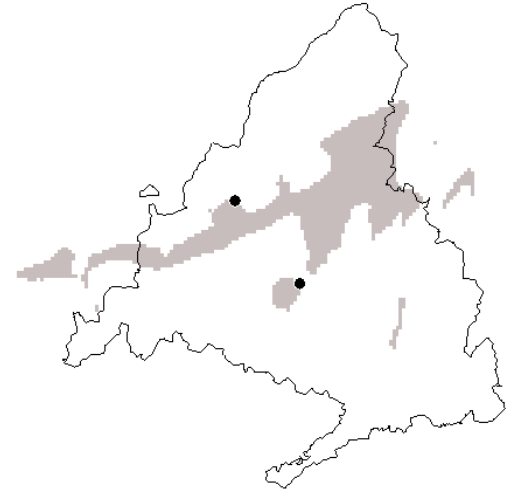

Aphodius (Melinopterus) abeillei

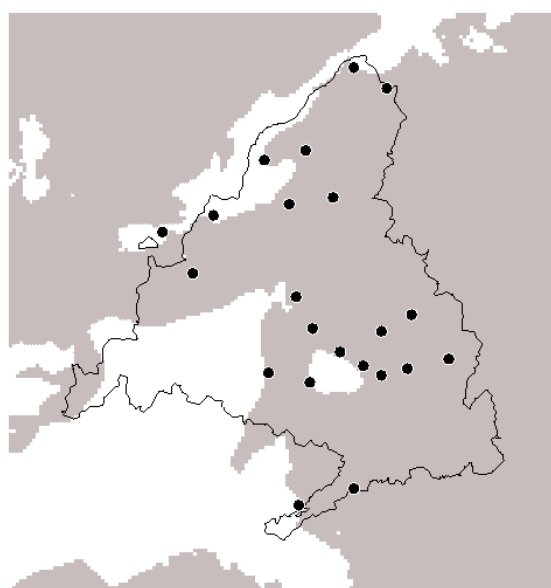

Aphodius (Melinopterus) prodromus

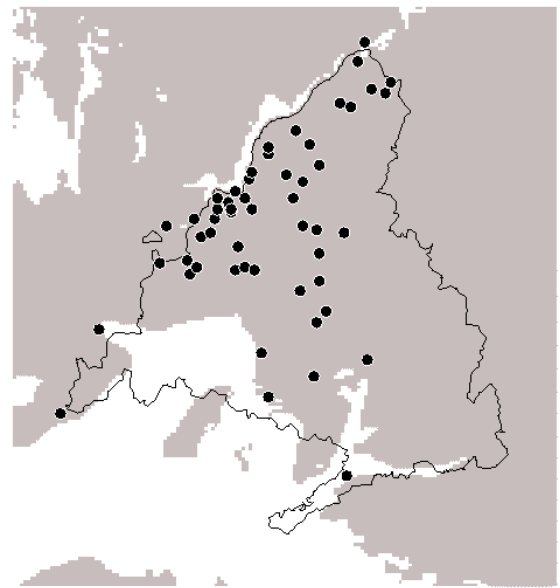

Aphodius (Melinopterus) sphacelatus

Fig. 1.- Cont 


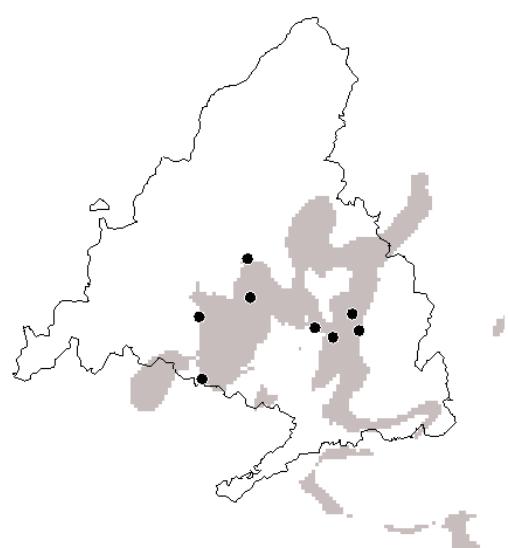

Aphodius (Melinopterus) tingens

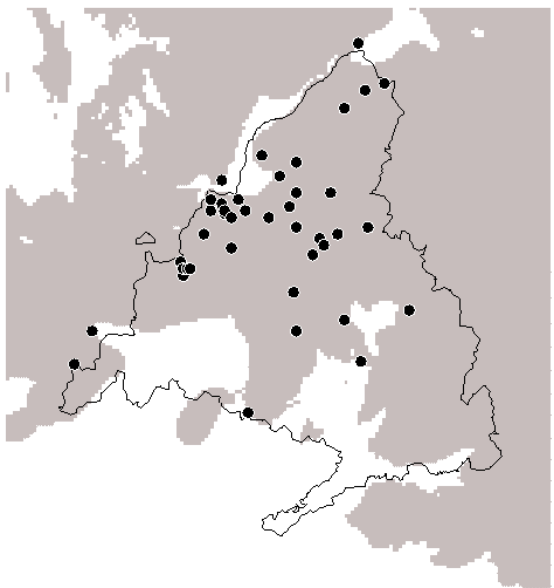

Aphodius (Nimbus) affinis

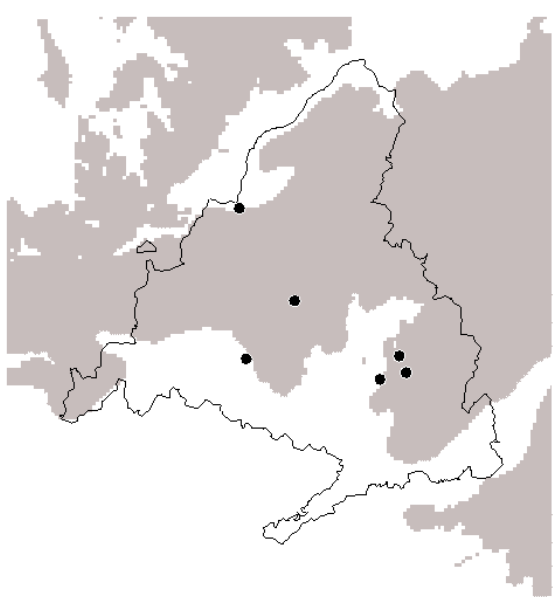

Aphodius (Nobius) bonnairei

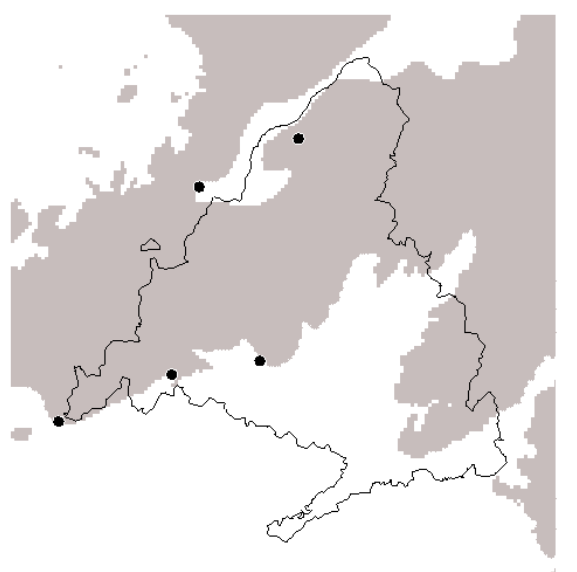

Aphodius (Nialus) varians

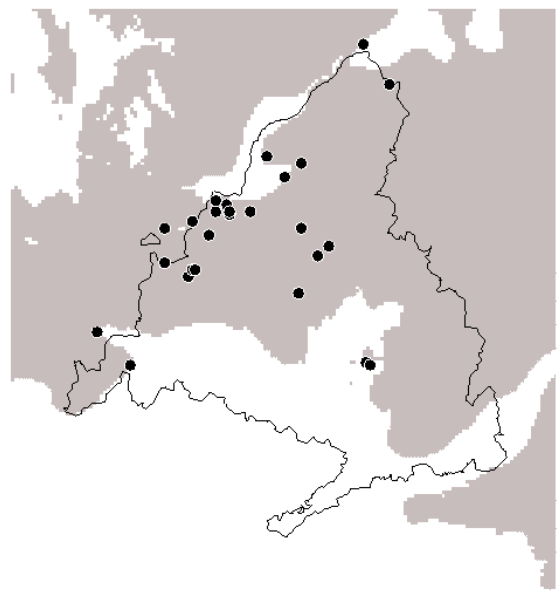

Aphodius (Nimbus) contaminatus

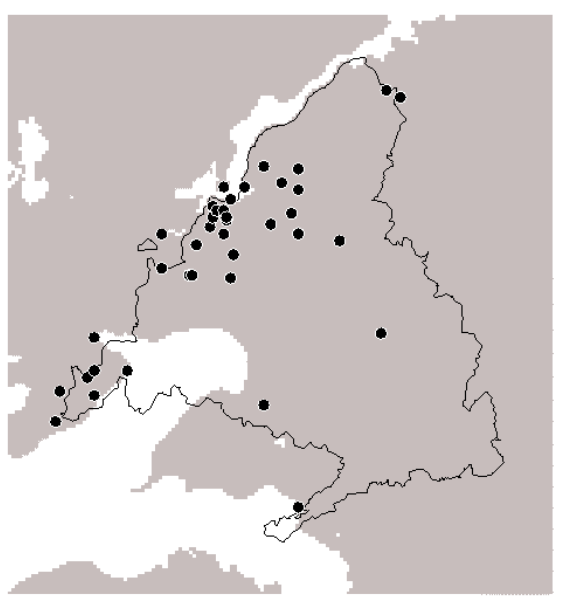

Aphodius ( Otophorus) haemorrhoidalis

Fig. 1.- Cont. 

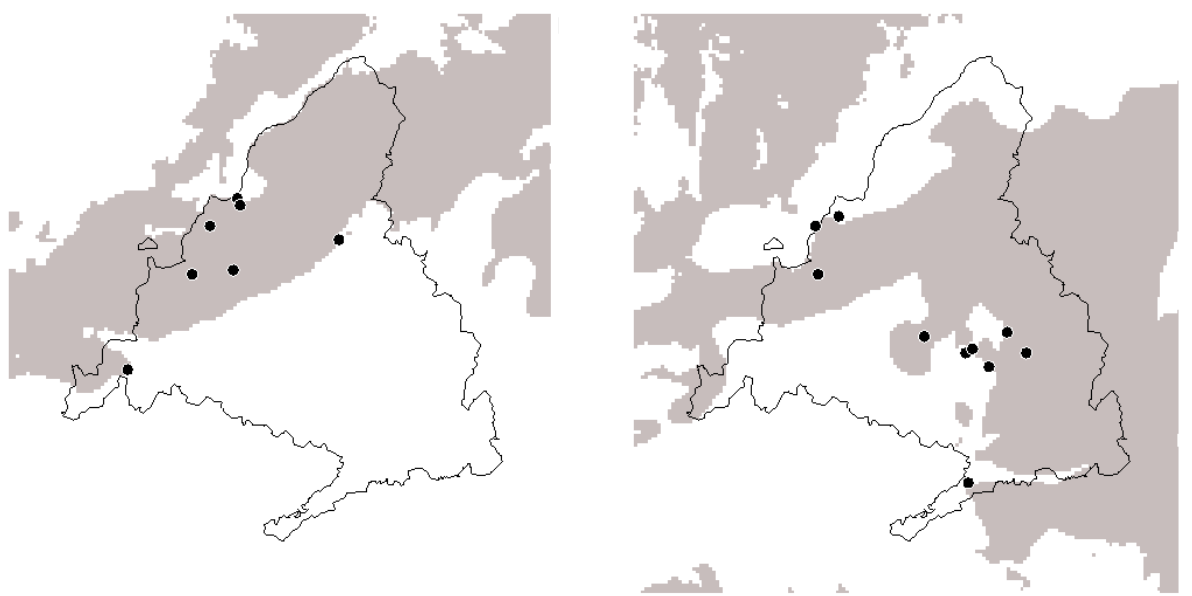

Aphodius (Phalacronothus) putoni Aphodius (Phalacronothus) quadrimaculatus

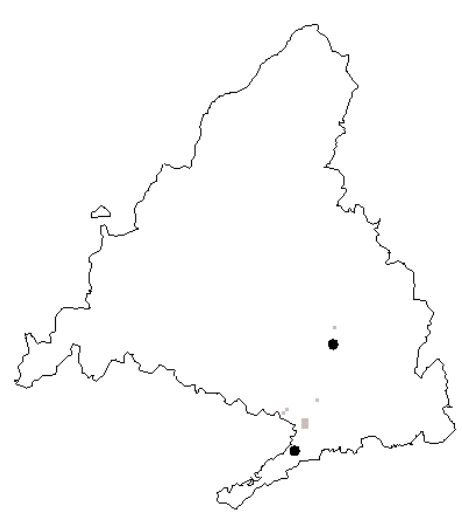

Aphodius (Plagiogonus) nanus

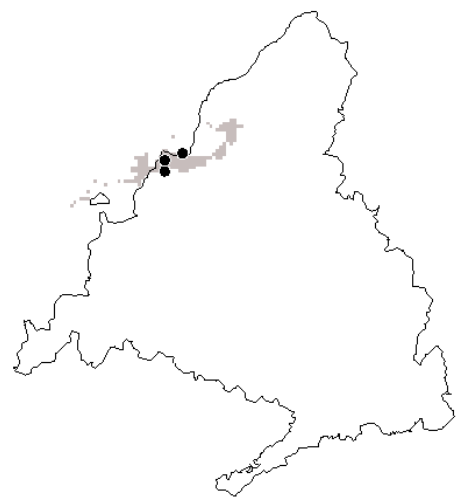

Aphodius ( Planolinus) uliginosus

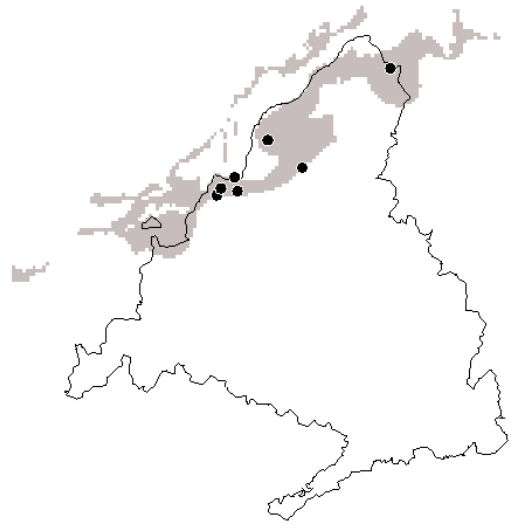

Aphodius (Planolinus) borealis

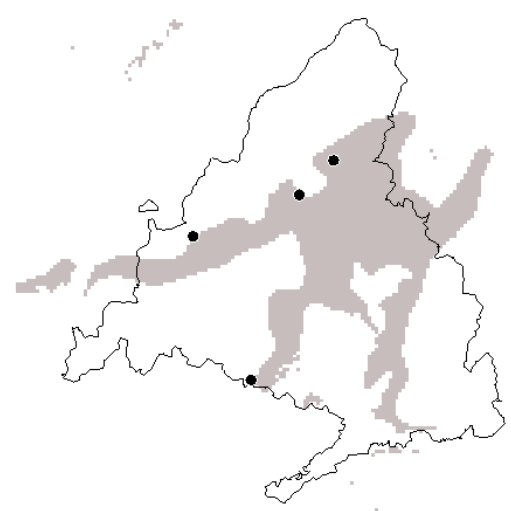

Aphodius ( Sigorus) porcus

Fig. 1.- Cont 


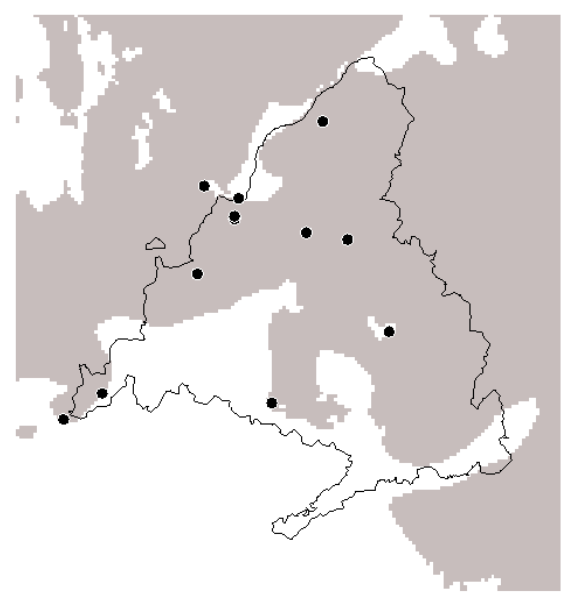

Aphodius (Subrinus) sturmi

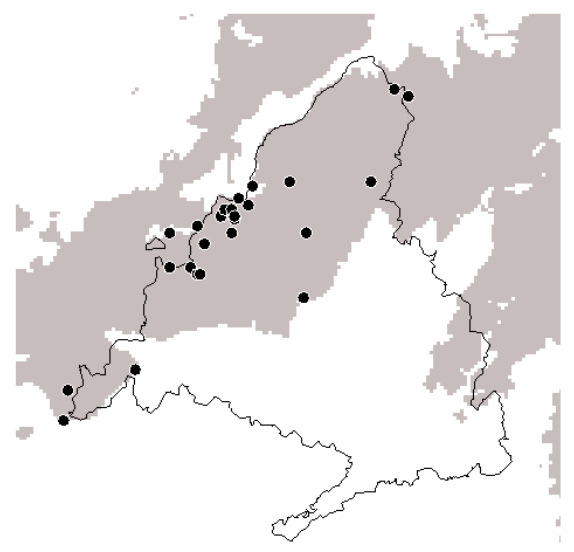

Aphodius (Trichonotulus) scrofa

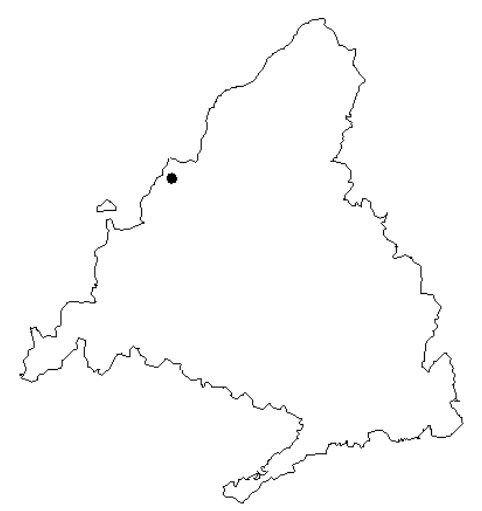

Platytomus tibialis

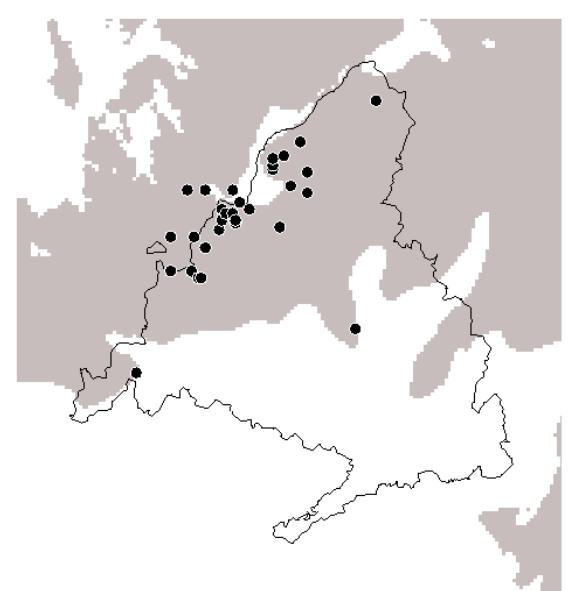

Aphodius (Teuchestes) fossor

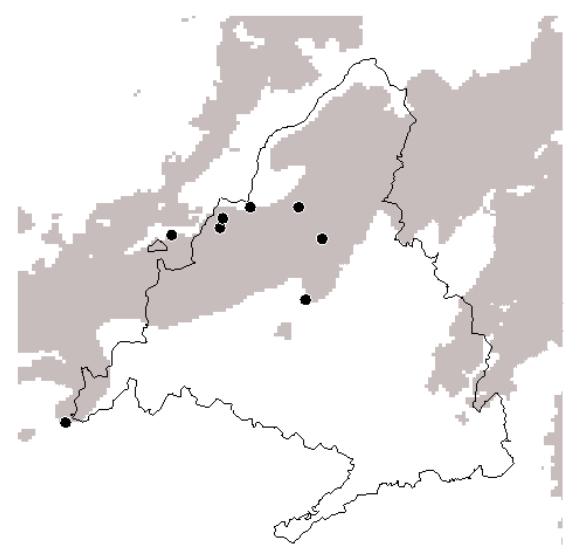

Heptaulacus testudinarius

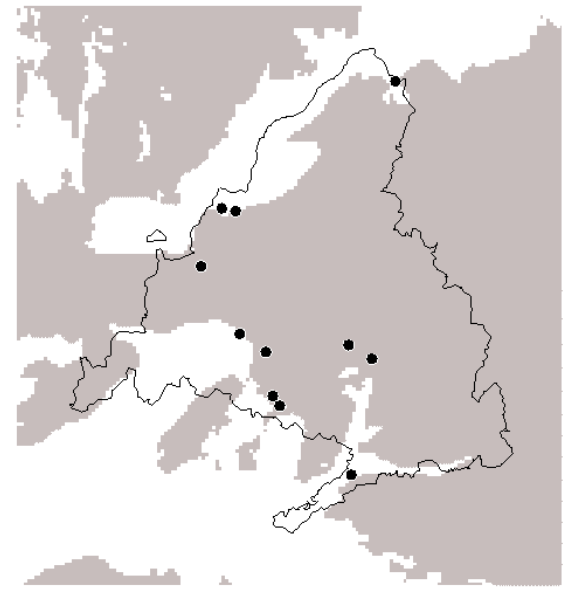

Pleurophorus caesus

Fig. 1.- Cont. 


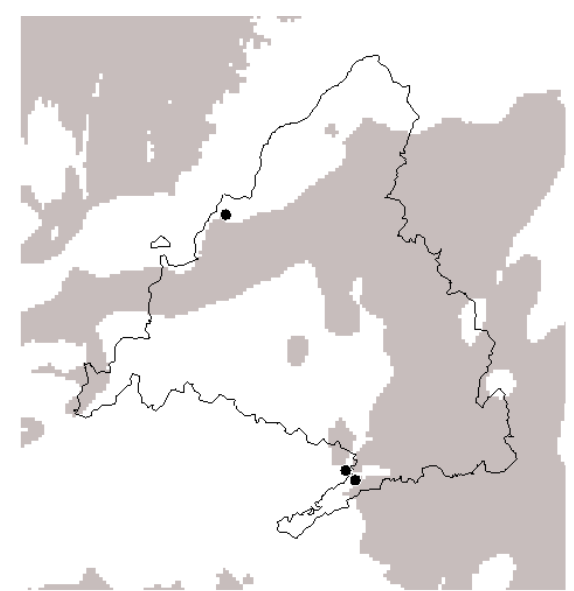

Pleurophorus mediterranicus

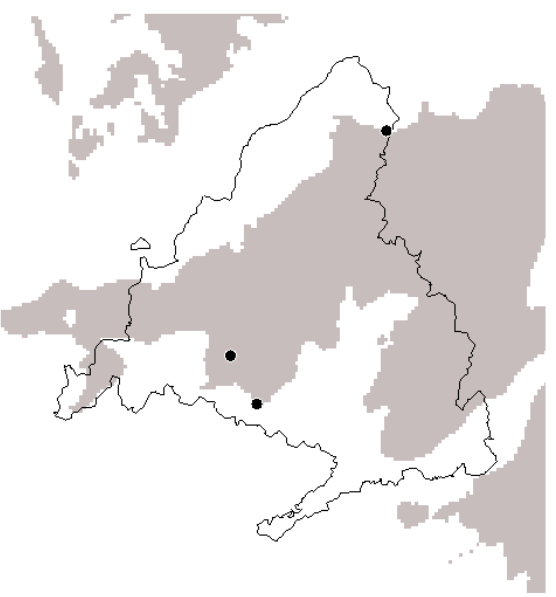

Rhyssemus algiricus

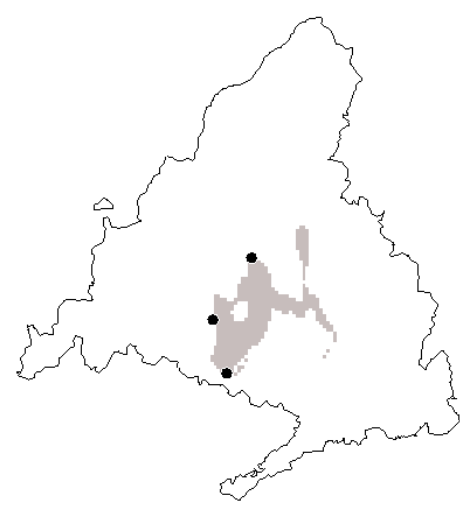

Psammodius (psammodius) laevipennis

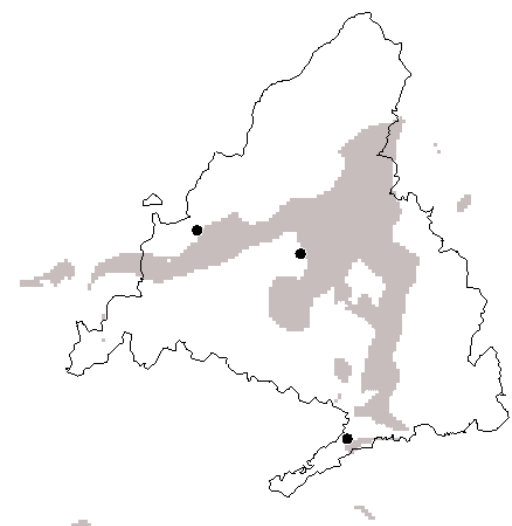

Rhyssemus germanus

Fig. 1.-Fin.

geográfica de la biodiversidad de escarabeidos coprófagos en Europa occidental (Lobo \& MartínPiera, 2002; Hortal et al., 2001, 2003; Lobo et al. 2002, 2004; Verdú \& Galante, 2002; Martín-Piera \& Lobo, 2003; Cabrero-Sañudo \& Lobo, 2003). En general, los efectos más importantes parecen provenir de las variables climáticas y de los factores de índole histórico-geográfica. Las variaciones climáticas tienen un importante efecto sobre la riqueza de los escarabeidos en general, lo que evidencia un probable control climático sobre los ensamblajes de Scarabaeoidea, resultado de sus limitaciones y requerimientos ambientales. La importancia de los factores histórico-geográficos se traduce en una importante modificación del patrón o estructura espacial producido por el efecto de las variaciones ambientales (ver discusión en Hortal et al., 2001; Lobo \& Martín-Piera, 2002; Hortal \& Lobo, 2001, 2002), debido a los procesos históricos de dispersión y/o extinción. Litología, heterogeneidad ambiental y calidad del recurso trófico aparecen como factores secundarios en el caso de las variaciones en la riqueza (Cabrero-Sañudo \& Lobo, 2003) o la composición faunística (Hortal et al., 2003). En el caso de la endemicidad, la existencia de una fauna estrechamente adaptada al consumo de las heces con bajo contenido hídrico como las de conejo, parece ser la causa principal de su variación Ibérica (Verdú \& Galante, 2002).

Una buena parte de las especies de afódidos de la Comunidad de Madrid presentan adaptaciones al clima frío y húmedo de las áreas montañosas. 

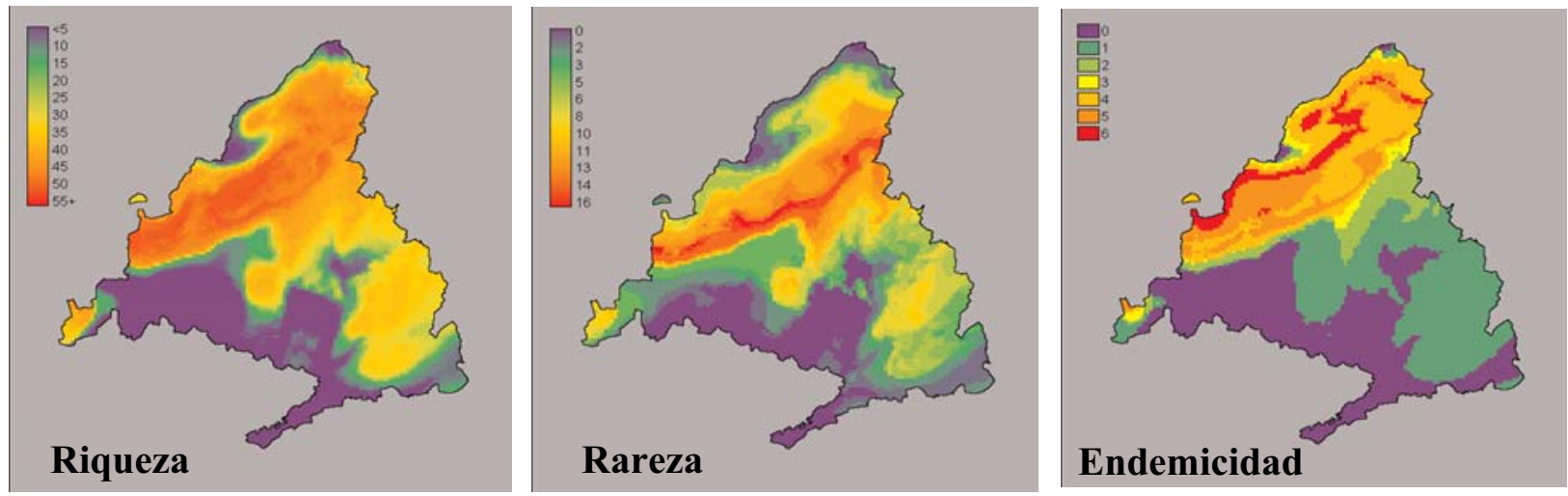

Fig. 2.- Mapas de riqueza (número de especies), rareza (número de especies raras) y endemicidad (número de endemismos ibéricos) potencial de afódidos en Madrid y alrededores. Cada mapa se ha obtenido mediante la superposición de las distribuciones potenciales anteriormente calculadas para todas las especies, las especies raras, y las especies endémicas, respectivamente.

Fig. 2.- Maps of potential richness (number of species), rarity (number of rare species) and endemism (number of Iberian endemics) of Aphodiidae at Madrid and the surrounding areas. Each map was obtained overlying the potential distributions formerly calculated for all species, rare species, and endemic species, respectively.

Cuando la variación de las comunidades se analiza a lo largo de un gradiente ambiental como el que aparece altitudinal o latitudinalmente, aparece un claro relevo faunístico ente los Scarabaeidae, que dominan en las comunidades templado-cálidas y de baja altitud, y los Aphodiinae, que lo hacen bajo condiciones frías y de altura. Esta segregación espacial ha sido atribuida a la influencia de factores de tipo histórico y a la persistencia de adaptaciones eco-fisiológicas ancestrales hacia distintas condiciones ambientales en ambos linajes, originados en regiones biogeográficas diferentes (Martín-Piera et al., 1992; Lobo 1992b, 1997; Jay-Robert et al., 1997; Lobo \& Halffter, 2000). A pesar de presentar ciertos elementos de origen Afrotropical, muchas de las especies Ibéricas de afódidos presentan adaptaciones templado-frías, son de origen Eurosiberiano o Laurásico y podrían haber colonizado la Península Ibérica durante las épocas glaciares atravesando los Pirineos por su parte occidental para colonizar la Cordillera Cantábrica, el norte de Portugal e incluso el Sistema Central. La existencia de un claro gradiente longitudinal independiente de las condiciones ambientales avala este supuesto (Cabrero-Sañudo \& Lobo, 2003; Lobo et al., 2004). La distribución potencial de las especies de este gran componente Eurosiberiano se superpone con la de algunas otras especies Afrotropicales que poseen en estas mismas zonas madrileñas su límite de distribución septentrional, motivo por el cual riqueza y rareza son mayores en las zonas de altitud media. Sin embargo, dado el origen Eurosiberiano de las especies de afódidos adaptadas a los ambientes de montaña, resulta ilustrativo reconocer que la mayor parte de endemismos ibéricos presentes en la Comunidad de Madrid son, también, especies de montaña. Algunos de estos endemismos, como Aphodius (Acrossus) carpetanus, Aphodius (Agolius) bonvoulouri o Aphodius (Ammoecius) frigidus, podrían ser en realidad neoendemismos, especies que tuvieron rangos de distribución más amplios durante las pasadas glaciaciones y que, actualmente, se encuentran refugiadas en la Península Ibérica (Cabrero-Sañudo \& Lobo, 2003). En general, las adaptaciones frías del fuerte componente Eurosiberiano que colonizó la Península Ibérica, tal vez durante la última glaciación, están detrás de los patrones de diversidad potencial de este grupo en Madrid, constituyendo un buen ejemplo de la influencia de los factores histórico-evolutivos en los patrones actuales de variación de la diversidad biológica.

\section{AGRADECIMIENTOS}

Este trabajo no habría sido posible sin la ayuda de Francisco Cabrero y Fermín Martín Piera. Agradecemos también a Miguel Corra y a Juan Jesús de la Rosa que nos hayan permitido acceder a sus colecciones privadas. Trabajo financiado por el proyecto GR/AMB/0750/2004 de la Comunidad de Madrid. Se ha obtenido financiación adicional del proyecto de la Fundación BBVA "Yámana - Diseño de una red de reservas para la pro- 
tección de la biodiversidad en América del Sur Austral utilizando modelos predictivos de distribución con taxones hiperdiversos", así como del proyecto CGL2004-0439/BOS del Ministerio de Educación y Ciencia. JH disfruta de una beca de la FCT (Fundação para a Ciência e Tecnologia) de Portugal (BPD/20809/2004).

\section{Referencias}

Allsopp, P. G., 1999. How localized are the distributions of Australian scarabs (Coleoptera, Scarabaeoidea). Diversity and Distributions, 5: 143-149.

BARAUD, J., 1992. Coléoptères Scarabaeoidea d'Europe. Féderation Française des Sociétés de Sciences Naturelles. Lyon. 856 pp.

Brown, J. H. \& Lomolino, M. V., 1998. Biogeography. Second edition. Sinauer Associates Inc. Sunderland. $691 \mathrm{pp}$.

Browne, J. \& Scholtz, C. H., 1999. A phylogeny of the families of Scarabaeoidea (Coleoptera). Systematic Entomology, 24: 51-84.

BUSBY, J.R. 1986. A biogeoclimatic analysis of Notophagus cunninghamii (Hook.) Oerst. in southeastern Australia. Australian Journal of Ecology, 11: $1-7$

Cabrero-Sañudo, F. J., 2004. Análisis filogenético de los Aphodiinae (Coleoptera, Scarabaeoidea) ibéricos. Composición faunística, distribución y diversidad de especies en la Península Ibérica. Tesis Doctoral, Universidad Complutense de Madrid. $555 \mathrm{pp}$.

Cabrero-Sañudo, F. J. \& Lobo, J. M., 2003. Reconocimiento de los factores determinantes de la riqueza de especies: El caso de los Aphodiinae (Coleoptera, Scarabaeoidea, Aphodiidae) en la Península Ibérica. Graellsia, 59: 155-177.

CABrero-Sañudo, F. J. \& Zardoya, R., 2004. Phylogenetic relationships of Iberian Aphodiini (Coleoptera: Scarabaeidae) based on morphological and molecular data. Molecular Phylogenetics and Evolution, 31(3): 1084-1110.

CAmBefort, Y., 1991. From Saprophagy to Coprofagy. In: I. Hanski \& Y. Cambefort (eds.). Dung Beetle Ecology. Princeton University Press. New Jersey: 51-68.

CAmbefort, Y. \& HansKi, I., 1991. Dung Beetle Population Biology. In: I. Hanski \& Y. Cambefort (eds.). Dung Beetle Ecology. Princeton University Press. New Jersey: 36-50.

Clark LaBS, 2003. Idrisi Kilimanjaro. GIS software package. Clark Labs. Worcester.

Dellacasa, G., Bordat, P. \& Dellacasa, M., 2000. A revisional essay of world genus-group taxa of Aphodiinae (Coleoptera Aphodiidae). Memorie della Società Entomologica Italiana, 79: 1-482.
DouBE, B. M., 1990. A functional classification for analysis of the structure of dung beetle assemblages. Ecological Entomology, 15: 371-383.

Gaston, K. J., 1994. Rarity. Chapman \& May. London. $220 \mathrm{pp}$.

Gaston, K. J., 1996. What is biodiversity? In: K. J. Gaston (ed.). Biodiversity. A Biology of Numbers and Difference. Blackwell Science. Oxford: 1-9.

GonzÁlez-Megías, A. \& SÁnchez-Piñero, F., 2003. Effects of brood parasitism on host reproductive success: evidence from larval interactions among dung beetles. Oecologia, 134: 195-202.

Halffter, G. \& Edmonds, W. D., 1982. The Nesting Behaviour of Dung Beetles. An Ecological and Evolutive Approach. Instituto de Ecología, MABUNESCO. México, D. F. 176 pp.

Halffter, G. \& Matthews, E. G., 1966. The natural history of dung beetles of the subfamily Scarabaeinae (Coleoptera, Scarabaeidae). Folia Entomologica Mexicana, 12-14: 1-312.

HANSKI, I., 1987. Nutritional ecology of dung and carrion-feeding insects. In: F. Slansky \& J. G. Rodríguez (eds.). Nutritional ecology of insects, mites, and spiders. John Wiley \& Sons. New York: 837-884.

Hortal, J., 2004. Selección y Diseño de Áreas Prioritarias de Conservación de la Biodiversidad mediante Sinecología. Inventario y modelización predictiva de la distribución de los escarabeidos coprófagos (Coleoptera, scarabaeoidea) de Madrid. Tesis Doctoral, Universidad Autónoma de Madrid. $333 \mathrm{pp}$.

Hortal, J., Cabrero-Sañudo, F. J. \& de la Rosa, J. J., 2002. Discusión sobre las especies de Liothorax (Coleoptera, Aphodiinae) en la Península Ibérica. Nueva cita para Ciudad Real de Liothorax cylindricus (Reiche \& Saulcy, 1856). Boletín de la Sociedad Entomológica Aragonesa, 31: 111-112.

Hortal, J. \& LoBO, J. M., 2001. A preliminary methodological approach to model the spatial distribution of biodiversity attributes. In: J. Mateu \& F. Montes (eds.). Spatio-temporal modelling of environmental processes. Proceedings of the $1^{\text {st }}$ Spanish workshop on spatio-temporal modelling of environmental processes. Publicacions de la Universitat Jaume I. Castelló de la Plana: 211-229.

HorTal, J. \& Lobo, J. M., 2002. Una metodología para predecir la distribución espacial de la diversidad biológica. Ecología (n.s.), 16: 151-178 + 114 figures.

Hortal, J. \& Lobo, J. M., 2005. An ED-based protocol for the optimal sampling of biodiversity. Biodiversity and Conservation, 14: 2913-2947.

Hortal, J., Lobo, J. M. \& Martín-Piera, F., 2001. Forecasting insect species richness scores in poorly surveyed territories: the case of the Portuguese dung 
beetles (Col. Scarabaeinae). Biodiversity and Conservation, 10: 1343-1367.

Hortal, J., Lobo, J. M. \& Martín-Piera, F., 2003. Una estrategia para obtener regionalizaciones bióticas fiables a partir de datos incompletos: el caso de los Escarabeidos (Coleoptera) Ibérico-Baleares. Graellsia, 59: 331-344.

Jay-Robert, P., Lobo, J. M. \& Lumaret, J. P., 1997. Altitudinal turnover and species richness variation in European montane dung beetle assemblages. Arctic and Alpine Research, 29: 196-205.

Loво, J. M., 1997. Influencias geográficas, históricas y filogenéticas sobre la diversidad de las comunidades locales: una revisión y algunos ejemplos utilizando Scarabaeoidea coprófagos (Coleoptera, Laparosciti). Boletín de la Asociación Española de Entomología, 21: 15-31.

LoBo, J. M., 1992a. Los Escarabeidos Coprófagos: un grupo de insectos con posibilidades. Zapateri, 1: 7378.

Loвo, J. M., 1992b. Modificación de las comunidades de Scarabaeoidea coprófagos (Coleoptera) en pastizales de altura del Sistema Central Ibérico (España) a lo largo de un gradiente altitudinal. Acta Zoologica Mexicana, 53: 15-31.

Lobo, J. M. \& HAlfFter, G., 2000. Biogeographical and ecologic factors affecting the altitudinal variation of mountainous communities of coprophagous beetles (Coleoptera: Scarabaeoidea): A comparative study. Annals of the Entomological Society of America, 93: 115-126.

Lobo, J. M., Jay-Robert, P. \& Lumaret, J. P., 2004. Modelling the species richness distribution for French Aphodiidae (Coleoptera, Scarabaeoidea). Ecography, 27: 145-156.

Lobo, J. M., Lumaret, J. P. \& JAY-Robert, P., 2002. Modelling the species richness of French dung beetles (Coleoptera, Scarabaeidae) and delimiting the predicitive capacity of different groups of explanatory variables. Global Ecology and Biogeography, 11: 265-277.

Lobo, J. M. \& Martín-Piera, F., 2002. Searching for a predictive model for species richness of Iberian dung beetle based on spatial and environmental variables. Conservation Biology, 16: 158-173.

López-Colón, J. I., 2000. Familia Geotrupidae. In: F. Martín-Piera \& J. I. López-Colón. Coleoptera, Scarabaeoidea I. Museo Nacional de Ciencias Naturales, Consejo Superior de Investigaciones Científicas. Madrid: 107-183.

Lumaret, J. P. \& LoBo, J. M., 1996. Geographic distribution of endemic dung beetles (Coleoptera, Scarabaeoidea) in the Western Palaearctic region. Biodiversity Letters, 3: 192-199.
Martín-PIERA, F., 2000a. Superfamilia Scarabaeoidea. Introducción General. In: F. Martín-Piera \& J. I. López-Colón. Coleoptera, Scarabaeoidea I. Museo Nacional de Ciencias Naturales, Consejo Superior de Investigaciones Científicas. Madrid: 15-41.

Martín-Piera, F., 2000b. Familia Scarabaeidae. In: F. Martín-Piera \& J. I. López-Colón. Coleoptera, Scarabaeoidea I. Museo Nacional de Ciencias Naturales, Consejo Superior de Investigaciones Científicas. Madrid: 207-432.

Martín-Piera, F. \& Lobo, J. M., 1993. New data and observations on kleptoparasitic behaviour in dung beetles from temperate regions (Coleoptera: Scarabaeoidea). Acta Zoologica Mexicana (nueva serie), 57: 15-18.

Martín-Piera, F. \& Lobo, J. M., 1995. Diversity and ecological role of dung beetles in Iberian grassland biomes. In: D. I. McCracken, E. M. Bignal \& S. E. Wenlock (eds.). Farming on the edge: the nature of traditional farmland in Europe. Joint Nature Conservation Comittee. Peterborough: 147-153.

Martín-Piera, F. \& Lobo, J. M., 2003. Database records as a sampling effort surrogate to predict spatial distribution of insects in either poorly or unevenly surveyed areas. Acta Entomológica Ibérica e Macaronésica, 1: 23-35.

Martín-Piera, F., Veiga, C. M. \& Lobo, J. M., 1992. Ecology and biogeography of dung-beetle communities (Coleoptera, Scarabaeoidea) in an Iberian mountain range. Journal of Biogeography, 19: 677-691.

Rabinowitz, D., Cairns, S. \& Dillon, T., 1986. Seven forms of rarity and their frequency in the flora of the British Isles. In: M. E. Soulé (ed.). Conservation Biology. The Science of Scarcity and Diversity. Sinauer Associates Inc. Sunderland: 182-204.

Ridsill-Smith, T. J. \& KIRK, A. A., 1981. Dung beetles and dispersal of cattle dung. Proceedings of the Australian Conference on Grassland Invertebrate Ecology, 3: 215-219.

RoJewski, C., 1983. Observations of the nesting behaviour of Aphodius erraticus (L.) (Coleoptera, Scarabeidae). Bulletin of Entomology Pologne, 53: 271-279.

TORIBIO, M., 1985. Los Cerambycidae y Scarabaeidae de Montebatres en el término municipal de Batres (Madrid) (Coleoptera). Boletín del Grupo Entomológico de Madrid, 1: 129-141.

Veiga, C. M., 1998. Los Aphodiinae (Coleoptera, Aphodiidae) Ibéricos. Tesis Doctoral, Universidad Complutense de Madrid. 573 pp. + apéndices.

Verdú, J. R. \& Galante, E., 2002. Climatic stress, food availability and human activity as determinants of endemism patterns in the Mediterranean region: the case of dung beetles (Coleoptera, Scarabaeoidea) in 
the Iberian Peninsula. Diversity and Distributions, 8: 259-274.

Verdú, J. R., Galante, E., Lumaret, J. P. \& CABrero-

SAÑUdo, F. J., 2004. Phylogenetic analysis of Geotrupidae (Coleoptera, Scarabaeoidea) based on larvae. Systematic Entomology, 29: 509-523.
Villalba, S., Lobo, J. M., Martín-Piera, F. \& ZARDOYA, R., 2002. Phylogenetic relationships of Iberian dung beetles (Coleoptera: Scarabaeinae): Insights on the evolution of nesting behaviour. Journal of Molecular Evolution, 55: 116-126. 